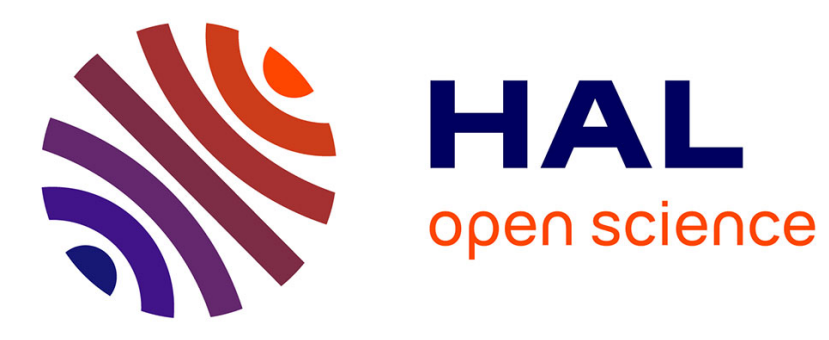

\title{
IXV post-flight reconstruction and analysis of the aerothermodynamic measurements along the rarefied portion of the reentry trajectory
}

\author{
Marc Schouler, Ysolde Prévereaud, Luc Mieussens
}

\section{- To cite this version:}

Marc Schouler, Ysolde Prévereaud, Luc Mieussens. IXV post-flight reconstruction and analysis of the aerothermodynamic measurements along the rarefied portion of the reentry trajectory. International Journal of Heat and Mass Transfer, 2021, 178, pp.121582. 10.1016/j.ijheatmasstransfer.2021.121582 . hal-03284302

\section{HAL Id: hal-03284302 \\ https://hal.science/hal-03284302}

Submitted on 12 Jul 2021

HAL is a multi-disciplinary open access archive for the deposit and dissemination of scientific research documents, whether they are published or not. The documents may come from teaching and research institutions in France or abroad, or from public or private research centers.
L'archive ouverte pluridisciplinaire HAL, est destinée au dépôt et à la diffusion de documents scientifiques de niveau recherche, publiés ou non, émanant des établissements d'enseignement et de recherche français ou étrangers, des laboratoires publics ou privés. 


\title{
IXV Post-flight Reconstruction and Analysis of the Aerothermodynamic Measurements along the Rarefied portion of the Reentry Trajectory
}

\author{
Marc Schouler $^{\mathrm{a}, *}$, Ysolde Prévereaud ${ }^{\mathrm{a}}$, Luc Mieussens $^{\mathrm{b}}$ \\ ${ }^{a}$ ONERA / DMPE, Université de Toulouse, F-31055 Toulouse, France \\ ${ }^{b}$ Bordeaux INP, Univ. Bordeaux, CNRS, IMB, UMR 5251, F-33400 Talence,France
}

\begin{abstract}
Following the reentry of the Intermediate eXperimental Vehicle in 2015, a lot of efforts were carried out at the ONERA for the post-flight analysis and the reconstruction of the heat flux applied to the vehicle. This flight represents a unique means of validation of the computational tools employed for the design of such mission. The aim of this paper is to exploit for the first time these data in the rarefied portion of the vehicle's reentry. In this context, DSMC simulations were performed between 115 and $90 \mathrm{~km}$ and a CFD computation was made for the $90 \mathrm{~km}$ flight point. In the standard numerical conditions, different degrees of agreement are obtained and a sensitivity analysis to various numerical and atmospheric parameters provides several ways for significantly improving the results. In addition, the DSMC and CFD comparison provides a good agreement for the surface quantities and suggestions to reach a better level of consistency between both methods and the flight data are finally discussed.
\end{abstract}

Keywords: Direct Simulation Monte-Carlo, Computational Fluid Dynamics, Rarefied hypersonic flow, Earth reentry, Experimental and flight data, Intermediate eXperimental Vehicle

\section{Introduction}

The experimental validation of the computational methods for fluid dynamics is a crucial step for the development of robust and reliable tools involved in the design of aerospace missions and vehicles. Carrying on-ground experiments able to recreate the higly energetic conditions characteristic of Earth atmospheric reentry is still, to this day, a challenging task and even more when it comes to low density flows [1, 2]. Hence, the performance of in flight experiments is the most valuable way of collecting all kind of data in these particular conditions.

Among the various reentry objects with which flight data were collected, we can mention the Japanese Orbital Reentry Experiment (OREX), the American Apollo module or the European Atmospheric Re-entry Demonstrator (ARD). In most cases, the carried instrumentation was designed for collecting data in the continuous portion of the reentry and was triggered after the passing of the entry point generally located at $120 \mathrm{~km}$. This is why even if most data were exploited for altitudes below $80 \mathrm{~km}$, some authors exploited the data collected during the transitional part of the reentry (generally above $85 \mathrm{~km}$ ) for comparison with highly nonequilibrium computational methods [3].

In February 2015, the Intermediate eXperimental Vehicle (IXV) was launched with a Vega launcher from French Guyana. After an overall flight of around $35000 \mathrm{~km}$ along its nominal trajectory, the vehicle finally successfully landed in the Pacific Ocean [4, 5]. The IXV was designed

*. Corresponding author

Email address: marc.schouler@onera.fr (Marc Schouler) 
as a reentry demonstrator in charge of performing a series of in-flight experiments. Among those experiments, the vehicle aimed at investigating the undergone aerothermodynamic (ATD) conditions during an atmospheric reentry thanks to a large set of thermocouples (TC) spread over the vehicle's windward. The exploitation of the TC measurements was the object of Van Ghele's PhD thesis [6] who developed and adapted an inverse method for the reconstruction of the convective heat flux applied to the IXV during its mission. Before that, pre-flight Direct Simulation MonteCarlo (DSMC) computations of the IXV reentry were performed by Banyai et al. [7], in order to investigate the aerodynamic behavior of the vehicle between 120 and $85 \mathrm{~km}$.

Our paper presents the post-flight exploitation of the aerothermodynamic data collected during the transitional portion of the IXV reentry. The reconstructed convective heat flux applied to the IXV is compared to an extensive series of simulations for altitudes between 115 and $90 \mathrm{~km}$, performed with the DSMC code SPARTA [8]. In addition to the direct comparison of the heat flux, the sensitivity to several DSMC parameters and models are investigated. Hence, a detailed study of the uncertainty in the free stream conditions, of the effects of collision model, of chemistry and of accommodation coefficients is made. Finally, a simulation of the IXV was performed with a Computational Fluid Dynamics (CFD) solver and is herein compared to the DSMC results, at an altitude of $90 \mathrm{~km}$.

This article is organized as follows. First, the IXV mission, the vehicle and its instrumentation as well as the flight conditions corresponding to the transitional portion of the reentry are described. Then, the two computational approaches are presented in section 3. The DSMC results are discussed in section 4 where the flowfield and surface quantities are discussed separately. In section 5, a particular attention is given to the comparison of DSMC and CFD methods at $90 \mathrm{~km}$. Again, the flowfield and the surface quantities are discussed separately in the light of DSMC/CFD references. In the last section, our conclusions are finally given.

\section{IXV mission}

The IXV mission was funded by the European Space Agency (ESA) with Thales Alenia Space Italy (TASI) as the project's prime contractor. The launch initially scheduled end of 2014 was finally postponed to 2015. The $11^{\text {th }}$ of February, a VEGA launcher departed French Guyana and injected the vehicle in an equatorial suborbital trajectory. The IXV joined the Entry Interface Point (EIP) at $120 \mathrm{~km}$ with a $7.44 \mathrm{~km} / \mathrm{s}$ velocity and followed a guided lifting entry of more than $7000 \mathrm{~km}$ as illustrated in Figure 1. For the last phase of the reentry, a 3 stage parachute system was deployed permitting the successful splashdown of the vehicle in the Pacific Ocean [4].

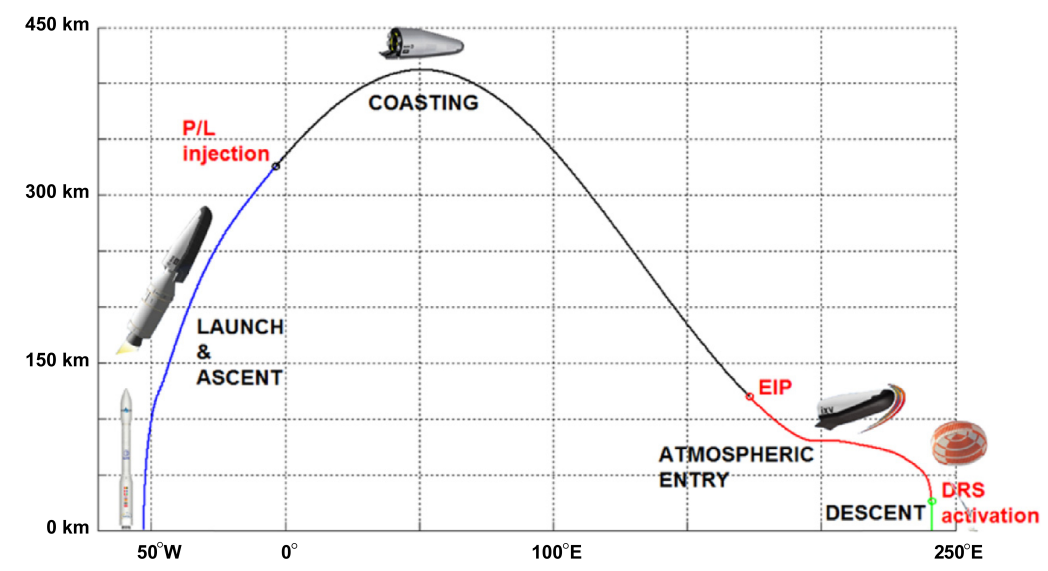

Figure 1: IXV reentry phases (from [4]).

Furthermore, as outlined by Van Ghele [6], the IXV trajectory is particularly close to some of the Space Transportation System (STS) mission trajectories. Hence, Figure 2 shows the simi- 
larities in trajectories which justify the analysis of the IXV flight data in the light of the STS measurements in spite of the great difference in the vehicles size.
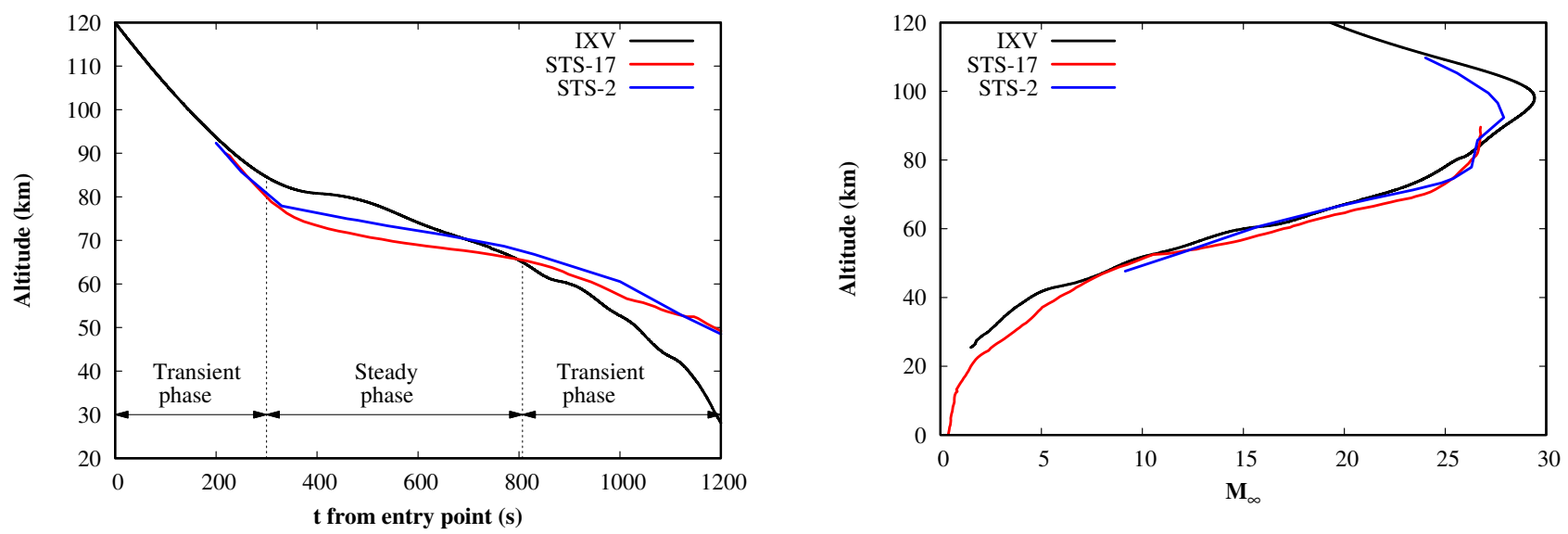

FIGURE 2: Comparison of IXV [9], STS-2 [10] and STS-17 [11] reentry trajectories : altitude as a function of time (left) and of upstream Mach number (right).

\subsection{IXV geometry}

The IXV aeroshape was designed by Dassault Aviation as a lifting body aerodynamically controlled by two body flaps. The smoothed geometry used for our computations is illustrated in Figure 3. As explained by Buffenoir et al. [12], the vehicle's Thermal Protection System (TPS) is

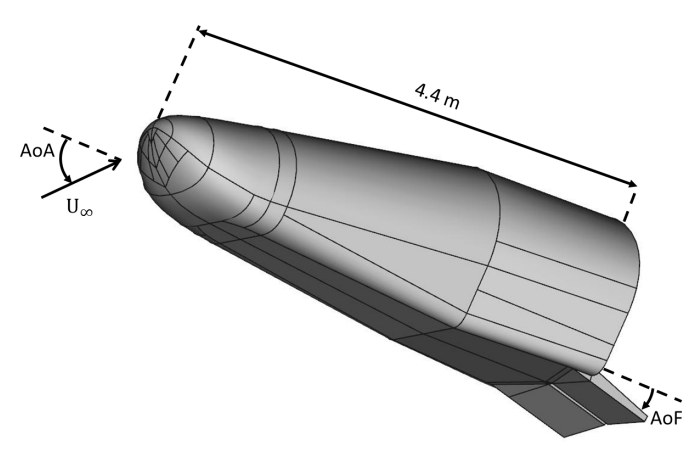

FIGURE 3: IXV smoothed geometry.

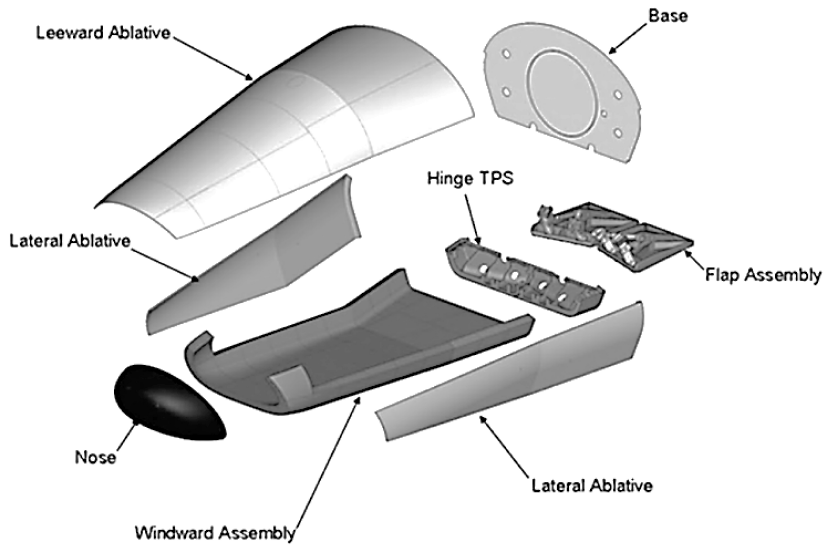

FIGURE 4: IXV TPS components (from [12]).

divided in an ablative and a non-ablative part (see Figure 4). The leeward, the lateral panels and the base assemblies are made of ablative materials whose conception, validation and integration steps are detailed in [13]. The remaining part of the TPS is based on a shingle design fulfilling separately a thermal and a mechanical function. Thus, a thin shell composed of a heat resistant Ceramic Matrix Composite (CMC) made of a Carbon-Silicium-Carbide (C-SiC) material is capable of maintaining the vehicle's aerodynamic line while withstanding the mechanical load resulting from the extreme heat flux. In the meantime, several layers of insulation located underneath the skin are in charge of absorbing the heat load to protect the cold structure from high temperatures. The TPS structure is finally anchored to the cold part thanks to an attachment system [12]. The windward comprises a total of 30 panels while the nose assembly is made of a unique monolithic C-SiC part presented in details in [14]. Finally, the hinge is also covered in a $\mathrm{C}-\mathrm{SiC}$ layer and the flaps are made of a Keraman C-SiC material described in [9]. 


\subsection{Flight instrumentation}

One of the main objectives of the IXV was to perform a series of in-flight experiments presented in [15]. The vehicle was instrumented accordingly to those experiments with around 300 sensors. Among them, 37 pressure sensors and 194 thermocouples (TC) comprising 105 type S platinum thermocouples and 89 type $\mathrm{K}$ thermocouples were integrated. In addition, the vehicle was equipped with 12 displacement sensors, 48 strain gauges and an Infra-Red (IR) camera system [15]. Among all the sensors and the investigated phenomena, two coated patches and 30 TC were used to study the catalytic behavior of the ceramic material. The evaluation of the shockwave boundary layer interaction was performed thanks to $21 \mathrm{TC}$ and 4 pressure sensors. The aerothermodynamic of the flaps was studied via the IR camera system together with TC. All the TC integrated to the windward were type $\mathrm{S}$ thermocouple capable of measuring temperatures between 273.15 and $1823.15 \mathrm{~K}$ with a $0.5 \%$ accuracy. The pressure sensing system was designed to withstand high temperatures and to be sensitive to pressures lower than $10 \mathrm{hPa}$. It was made of customized Kulite pressure transducers, pneumatic line and pressure ports [15]. However, since the pressure sensors were not designed to measure pressures as low as those encountered in the rarefied regime, their data turned out to be unusable. They were therefore not considered in our analysis.

For the present study, the measurements of the $10 \mathrm{TC}$ along the centerline as well as $10 \mathrm{TC}$ on the side of the vehicle were considered (see Figure 5). The inverse method used to reconstruct the convective heat flux from the temperature measurement is described in details in [6, 16]. In [16] the results of a sensitivity analysis showed that :

- During the transient phases of the flight (above $85 \mathrm{~km}$ and below $60 \mathrm{~km}$ - see Figure 2), the uncertainty of the $\mathrm{C}$-SiC layer thickness is the major source of uncertainty on the reconstructed heat flux.

- During the steady phase (between 85 and $60 \mathrm{~km}$ - see Figure 2), the major sources are the uncertainty of the emissivity value and of the surface temperatures.

Overall, the Monte-Carlo analyses reported in [16] indicates a maximum uncertainty smaller than $8 \%$ on the reconstructed heat flux all over the vehicle and for all the reentry.

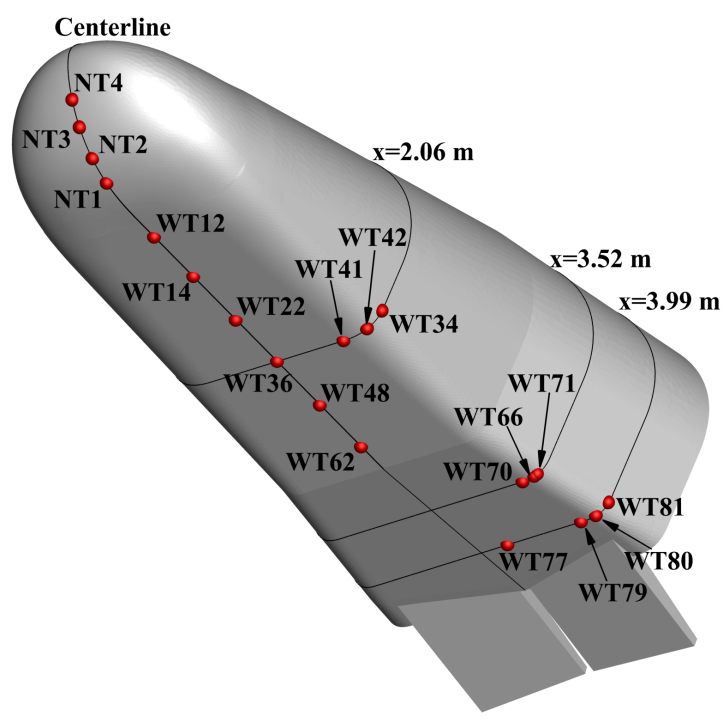

FiguRE 5: Thermocouples considered for the comparison of the numerical results and the flight data.

In addition to the instrumentation, the vehicle was also carrying a navigation system including an Inertial Measurement Unit (IMU) and a GPS receptor. The telecommunication network and 
the architecture of the in-flight experiment system in charge of recording and transmitting the data are respectively presented in [5] and [15].

\subsection{Flight parameters and atmospheric conditions}

The main flight parameters received from Thales Alenia Space Italy (TASI) are given in Table 1. These data show that for the investigated portion of the reentry, a $15^{\circ}$ flap inclination was

\begin{tabular}{cccccc}
\hline \hline $\mathrm{t}(\mathrm{s})$ & Alt. $(\mathrm{km})$ & $U_{\infty}(\mathrm{km} / \mathrm{s})$ & $\operatorname{AoA}\left(^{\circ}\right)$ & $\operatorname{AoS}\left(^{\circ}\right)$ & $\operatorname{AoF}\left(^{\circ}\right)$ \\
\hline 3933 & 115 & 7.44 & 46.96 & 0.31 & 15 \\
3968 & 110 & 7.45 & 45.45 & -0.98 & 15 \\
4005 & 105 & 7.46 & 44.51 & 0.34 & 15 \\
4045 & 100 & 7.46 & 46.12 & 0.59 & 15 \\
4087 & 95 & 7.46 & 46 & -0.09 & 15 \\
4134 & 90 & 7.46 & 46.94 & -0.46 & 15 \\
\hline \hline
\end{tabular}

AoA, AoS and AoF respectively denote the angle of attack, the sideslip angle and the flaps inclination.

TABLE 1: Flight parameters given by Thales Alenia Space Italy.

maintained. Moreover, because of the small sideslip angle $\left(<1^{\circ}\right)$, sideslip effects were simply neglected in our study.

In addition to those flight parameters, the exact longitude and latitude coordinates as a function of time were also provided by TASI. Hence, the knowledge of all those flight parameters enabled to fully determine the inputs of the US Naval Research Laboratory Mass Spectrometer and Incoherent Scatter radar Extended (NRLMSISE) model of 2000 (here referred as MSISE-00) and to take maximum advantage of the model. The corresponding atmospheric data are given in Table 2.

\begin{tabular}{cccccccc}
\hline \hline Alt. $(\mathrm{km})$ & $n_{\infty}\left(\cdot / \mathrm{m}^{3}\right)$ & $\rho_{\infty}\left(\mathrm{kg} / \mathrm{m}^{3}\right)$ & $T_{\infty}(\mathrm{K})$ & $\mathrm{X}_{\mathrm{O}_{2}}$ & $\mathrm{X}_{\mathrm{N}_{2}}$ & $\mathrm{X}_{\mathrm{O}}$ & $K n$ \\
\hline 115 & $6.97 \times 10^{17}$ & $3.08 \times 10^{-8}$ & 306 & 0.1066 & 0.7309 & 0.1625 & 0.43 \\
110 & $1.58 \times 10^{18}$ & $7.17 \times 10^{-8}$ & 235 & 0.1266 & 0.7517 & 0.1218 & 0.18 \\
105 & $4.29 \times 10^{18}$ & $1.99 \times 10^{-7}$ & 183 & 0.149 & 0.7704 & 0.0807 & 0.061 \\
100 & $1.25 \times 10^{19}$ & $5.91 \times 10^{-7}$ & 159 & 0.1703 & 0.7847 & 0.045 & 0.021 \\
95 & $3.37 \times 10^{19}$ & $1.62 \times 10^{-6}$ & 161 & 0.1865 & 0.7949 & 0.0186 & 0.0077 \\
90 & $8.12 \times 10^{19}$ & $3.92 \times 10^{-6}$ & 175 & 0.1966 & 0.7991 & 0.0043 & 0.0033 \\
\hline \hline
\end{tabular}

$n_{\infty}, \rho_{\infty}, T_{\infty},\left(\mathrm{X}_{\mathrm{O}_{2}}, \mathrm{X}_{\mathrm{N}_{2}}, \mathrm{X}_{\mathrm{O}}\right)$ are the free stream numerical density, mass density, temperature, species fraction and $K n$ is the Knudsen number computed with $\lambda_{\infty}^{\mathrm{VHS}}$ and $L_{\mathrm{ref}}=4.4 \mathrm{~m}$.

TABLE 2: Atmospheric conditions computed with the MSISE-00 model.

Even though a complete knowledge of the MSISE-00 model input parameters permits to alleviate the uncertainty related to them, an overall uncertainty on the density comprised between 10 and $15 \%$ is still estimated above $90 \mathrm{~km}[17,18]$. The last reference even mentions an uncertainty up to $100 \%$ in case of local and spontaneous atmospheric variations.

As it will be shown in section 4.3.5, a perfect knowledge of the atmospheric entry conditions is essential for a satisfactory reconstruction of the flight data in the rarefied regime since it helps to avoid significant uncertainties. However, this objective is not reachable at high altitudes with the standard atmospheric models currently available and is still challenging. 


\section{Computational approaches}

Numerical simulations were performed with the DSMC code SPARTA for the flight points given in Table 2. In order to have a better understanding of the differences between the DSMC results and the measurements, an additional computation was made with the ONERA CFD code CEDRE for the lowest flight point $(90 \mathrm{~km})$. This section introduces the numerical tools and parameters that were used for these computations.

\subsection{The DSMC computations}

In the conditions usually encountered at high altitudes where the density is low, the rate of inter-molecular collisions is not large enough to maintain the state of equilibrium of the gas. Ultimately, this leads to the failure of the classic continuum equations and gas kinetic based numerical methods must be used instead. Among the many computational approaches for the modeling of nonequilibrium flows, Bird's DSMC method emerged as one of the most used methods for the simulation of hypersonic rarefied flows encountered in atmospheric reentry. Indeed, this method provides a numerical solution of the Boltzmann equation which holds for any Knudsen number. For more details about the method, the reader is referred to the following books [19, 20] and articles [21, 22].

\subsubsection{Computational settings}

To ensure the physical reliability of any DSMC simulation, several numerical criteria or metrics must be met. In order to prevent particles to travel along distances greater than a mean free path, the time step must be smaller than the minimum mean free time in the domain. In the same way, in order to avoid particles to interact with other particles further than a mean free path, the cell size must be smaller than the local mean free path $\lambda_{\mathrm{x}, \mathrm{y}, \mathrm{z}}$. Finally, in order to obtain statistically reliable results, the number of numerical particles per cell must be greater than a certain number ${ }^{1}$ and the sampling procedure must be performed on a sufficient number of time steps. Accordingly to those criteria, we built our simulations as follows :

- The time step $\Delta t$ was chosen as a fraction of the minimum mean free time in the domain.

- The computational grid consists of a homogeneous Cartesian mesh of cell size $\Delta_{\mathrm{x}, \mathrm{y}, \mathrm{z}}$ equals to a fraction of the free stream mean free path $\lambda_{\infty}^{\mathrm{VHS}}$. In addition, a refinement procedure was used to split the cells where their size was found to be greater than the local mean free path (i.e. when $\left.\Delta_{\mathrm{x}, \mathrm{y}, \mathrm{z}}>\lambda_{\mathrm{x}, \mathrm{y}, \mathrm{z}}\right)$. This procedure is illustrated in the next section.

- The number of numerical particles $N_{p}$ was chosen so that each cell contains a minimum of 10 numerical particles in the free stream. In practice, the total number of particles is irrelevant to the simulation preciseness but it gives an idea of the computational load. The critical criterion is the number of particles per cell $N_{p / c}$ which is discussed in the next section.

- For each simulation, a similar approach to that proposed by Banyai et al. [7] was used. A characteristic time $\tau_{f}$ was defined as the duration needed for one particle traveling at mean speed to cross the domain. Then, the sampling process was started after five to ten $\tau_{f}$ and realized over five to ten $\tau_{f}$. To ensure the reduction of the statistical noise, the sampling parameters of the $100 \mathrm{~km}$ altitude case were also used for the altitudes above.

Finally, the computational domain was defined as a box large enough for free stream conditions to be found at the inlet and for supersonic conditions to be reached at the outlet. The surface mesh used for our computations is illustrated in Figure 6. When simulating a wide range of altitudes, the fineness of the surface mesh can be problematic since it accentuates the statistical noise of the surface properties when the domain grid size encompasses too many surfaces elements. Here

1. This number varies from one author to another but Banyai [7] recommends to have around 8 numerical particles in the free stream for a 5 air species gas flow. 
the choice was made to use the same very fine mesh for all flight points which is why significant oscillations are sometimes visible along the windward centerline. Nevertheless, these oscillations are numerical artifacts that can easily be strongly reduced by using a coarser mesh or by filtering the wall distribution (i.e. by averaging the value of an element with that of its neighbors). The convergence and consistency of the simulations are discussed in section 3.1.2.

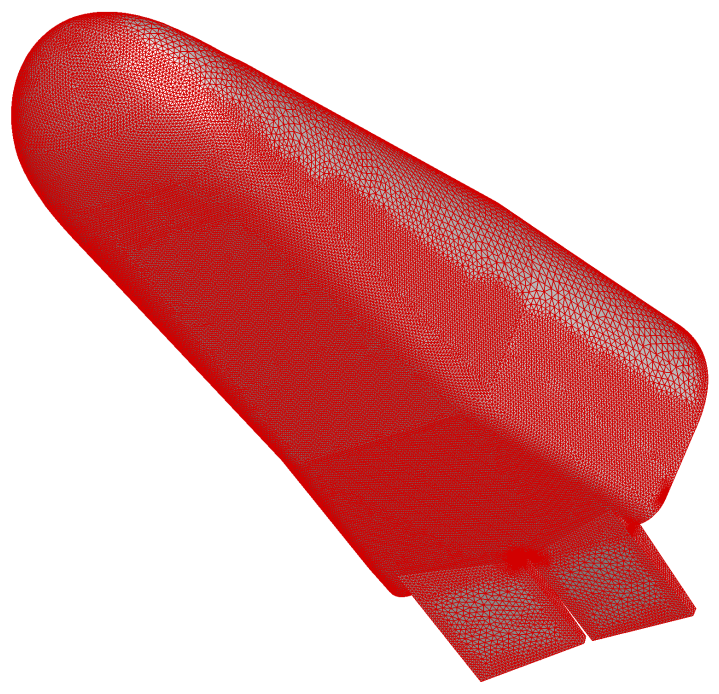

Figure 6: IXV Surface Mesh with 82726 elements.

Because of the vehicle's reentry velocity (around $7.5 \mathrm{~km} / \mathrm{s}$ ) and of the atmospheric density corresponding to the investigated altitudes, a five species air model was assumed and ionization effects were neglected. Among the physical models available in SPARTA, the Variable Hard Sphere (VHS) collision model was used with Bird's parameters (Appendix A of [19]) as the standard model for our study and the effect of this model was analyzed in depth via complementary computations. The No Time Counter (NTC) algorithm [20] was employed as the collision selection procedure. Both rotational and vibrational internal modes of energy were accounted for : the former as continuous with a constant relaxation number $Z_{\text {rot }}=5$, the latter as discrete with the Millikan-White variable relaxation number $Z_{\text {vib }}(T)$ defined in [19, 20]. Energy transfers were simulated with the Borgnakke-Larsen (BL) procedure and the default chemistry model employed was Bird's Total Collision Energy (TCE) model [19] with Park's kinetic coefficients. Dissociation and exchange reactions only were considered resulting in a set of 19 possible reactions (15 dissociation reactions, 2 direct-reverse exchange reactions). Moreover, since the considered TC are not located on the shingles covered with the catalytic coating, the wall was considered non-catalytic. Finally, the default gas surface interaction model was assumed fully diffuse with a constant wall temperature $T_{w}$ computed at each altitude as an average value of the measurements on the vehicle's windside (see Table 3). However, the effect of the accommodation coefficient was assessed for each altitude and various chemistry models were investigated at $90 \mathrm{~km}$. The influence of those parameters is discussed in section 4 .

\subsubsection{Time and space convergence}

The simulation parameters that were used for our computations are given in Table 3.

The table entries $\lambda_{\infty}^{\mathrm{VHS}} / \Delta_{\mathrm{x}, \mathrm{y}, \mathrm{z}}, \tau_{\text {coll }} / \Delta t, N_{p}$ and $T_{w}$ respectively denote the ratio between the free stream mean free path and the background mesh cell size, the ratio between the minimum mean collision time along the control line and the time step, the number of numerical particles 


\begin{tabular}{cccccc}
\hline \hline $\mathrm{t}(\mathrm{s})$ & Alt. $(\mathrm{km})$ & $\lambda_{\infty}^{\mathrm{VHS}} / \Delta_{\mathrm{x}, \mathrm{y}, \mathrm{z}}$ & $\tau_{\text {coll }} / \Delta t$ & $N_{p}$ & $T_{w}(\mathrm{~K})$ \\
\hline 3933 & 115 & 11 & 10 & $550 \times 10^{6}$ & 315 \\
3968 & 110 & 6 & 5.7 & $570 \times 10^{6}$ & 350 \\
4005 & 105 & 2.7 & 5.8 & $615 \times 10^{6}$ & 430 \\
4045 & 100 & 2.3 & 5 & $660 \times 10^{6}$ & 550 \\
4087 & 95 & 1.7 & 3.2 & $910 \times 10^{6}$ & 680 \\
4134 & 90 & 1 & 1.6 & $1400 \times 10^{6}$ & 870 \\
\hline \hline
\end{tabular}

TABLE 3: DSMC simulation parameters.

in the domain and the wall temperature. Considering the time step setting mentioned in the previous section, the ratio $\tau_{\text {coll }} / \Delta t$ sometimes falls below the critical value of 5 (Table 3 ). Hence, complementary simulations with time steps ensuring the respect of this criterion were performed for the 100, 95 and $90 \mathrm{~km}$ altitudes. The results of these computations showed no change in the heat flux and confirmed that all simulations whose results are presented herein are well resolved in time. With respect to the space convergence, a refinement procedure was enabled for all simulations. A SPARTA 3 level refinement procedure is shown in Figure 8. Such procedure limits to two the number of binary refinement in each direction (the first level corresponds to the background mesh). The cell size can be divided by two in each direction at each new level down to a grid size $\Delta_{\mathrm{x}, \mathrm{y}, \mathrm{z}} / 4$.

In order to assess the consistency of the numerical parameters, the values of interest were extracted along a control line as sketched in Figure 7. The results along the control line are expressed as a function of the distance from the NT3 thermocouple. For altitudes between 115 and $95 \mathrm{~km}$, the number of numerical particles per cell remains greater than 7 and $\lambda_{\mathrm{x}, \mathrm{y}, \mathrm{z}} / \Delta_{\mathrm{x}, \mathrm{y}, \mathrm{z}}$ reaches a minimum value of 0.9 for the $95 \mathrm{~km}$ case only in the cell adjacent to the surface. Between 115 and $100 \mathrm{~km}$, the $\lambda_{\mathrm{x}, \mathrm{y}, \mathrm{z}} / \Delta_{\mathrm{x}, \mathrm{y}, \mathrm{z}}$ ratio is strictly greater than 1 and the number of numerical particles is far greater than 10 all along the centerline.

For the $90 \mathrm{~km}$ altitude, the space and time criteria can be met but is computationally expensive since it requires a great amount of numerical particles, a very fine mesh and a small time step. Thus, to ensure that our parameter choice was appropriate, several simulation configurations were tested with various mesh domains and time step. The three domains are represented in Figure 9. The simulation configurations and maximum heat flux along the center line are provided in Table 4. The three heat flux along the centerline of the vehicle are given in Figure 10 and several values of interest along the control line are given in Figure 11. Figure 11a shows the increase in the numerical particle number per cell $\left(N_{p / c}\right)$ because of the new particles created through the dissociation process and the increase in the numerical density towards the wall. Figure $11 \mathrm{~b}$ illustrates the rise in the $\lambda_{x, y, z} / \Delta_{x, y, z}$ ratio up to a maximal value of around 2 for the run 1 and 2 , and twice as large for run 3 (background mesh twice as fine). In order to understand the nature of the $\lambda_{x, y, z} / \Delta_{x, y, z}$ ratio evolution, let us consider the expression of the local mean free path $\lambda_{x, y, z}$ used in this study :

$$
\lambda_{x, y, z}=\underbrace{\frac{1}{\sqrt{2 \pi d_{\mathrm{ref}, \mathrm{N}_{2}}^{2} n}}}_{(1)} \underbrace{\left(\frac{T}{T_{\mathrm{ref}, \mathrm{N}_{2}}}\right)^{\omega_{\mathrm{N}_{2}}-1 / 2}}_{(2)},
$$

where $d_{\text {ref, } \mathrm{N}_{2}}$ is the diameter of reference, $T_{\text {ref, } \mathrm{N}_{2}}$ is the temperature of reference and $\omega_{\mathrm{N}_{2}}$ is the viscosity index of $\mathrm{N}_{2}$. These VHS parameters are given in appendix of [19]. The consideration of the terms (1) and (2) facilitates the identification of the members respectively responsible for the decrease and the increase of the mean free path. Indeed, the increase in the temperature $T$ across the shock is such that the rise in term (2) overcomes the diminution of term (1). Thus, the 
$\lambda_{x, y, z} / \Delta_{x, y, z}$ ratio increases. As we get closer to the vehicle, the exponential rise in the density $n$ and the progressive diminution of the temperature slope and value results in the fall of the local mean free path down to a critical value $\lambda_{x, y, z} \leq \Delta_{x, y, z}$ which triggers the adaptive refinement. Between $x=0$ and $x=0.1 \mathrm{~m}$, Figure $11 \mathrm{~b}$ shows the significant oscillations of $\lambda_{x, y, z} / \Delta_{x, y, z}$ with 2 and 3 local minima corresponding to the different levels of adaptive refinement. Since the mesh density increases and the total number of numerical particles $N_{p}$ remains nearly constant, the refinement areas also correspond to the areas with a strong decrease of $N_{p / c}$ visible in Figure 11a.

According to the small variation of the wall heat flux along the centerline (maximum discrepancy smaller than 3\%), the configuration 1 was deemed acceptable and was then used for this study.

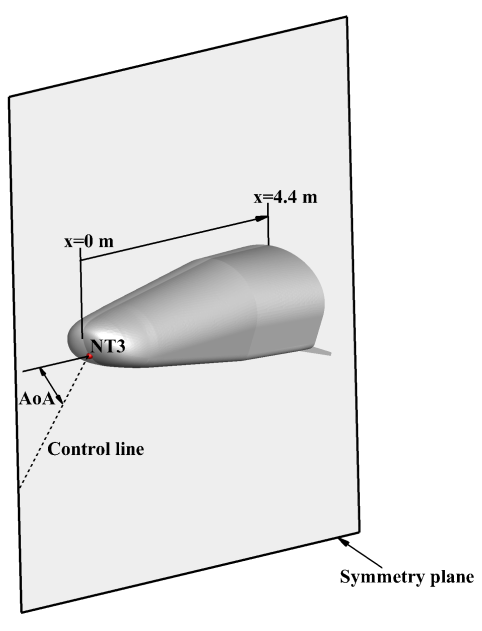

Figure 7: Extracted line for the control of the numerical criteria.

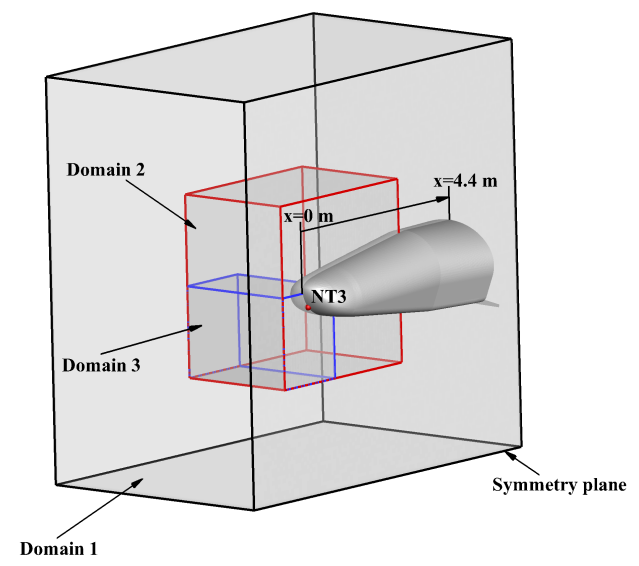

FiguRE 9: Various computational domains for the case at $90 \mathrm{~km}$.

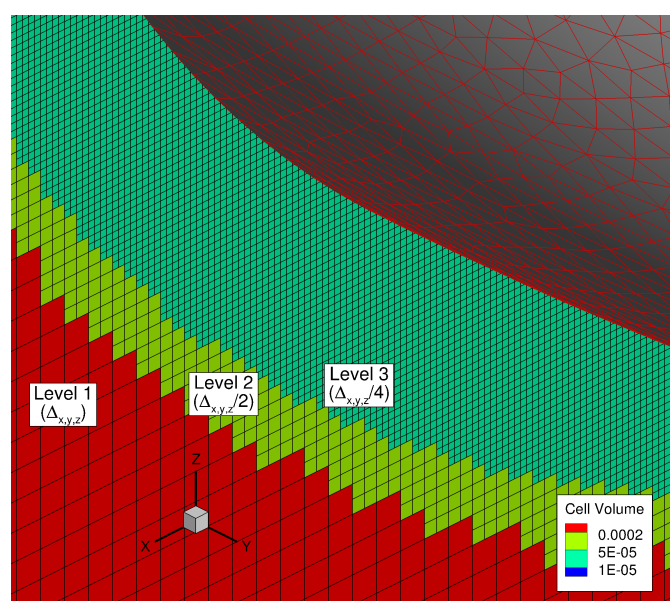

Figure 8: Schematic of a SPARTA 3 level refinement procedure at $90 \mathrm{~km}$.

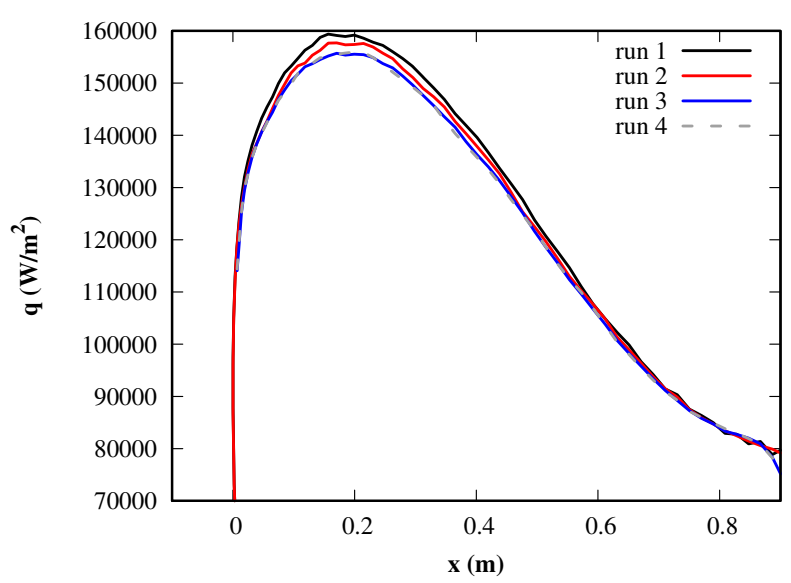

Figure 10: Comparison of the heat flux distribution along the vehicle windward centerline for four refinement configurations at $90 \mathrm{~km}$.

\subsection{The CFD computations}

\subsubsection{Mesh description}

For the CFD simulation, an unstructured mesh of 12.6 millions of elements, including prism and tetrahedron, was built with the ICEM software (see Figure 12) as follows. The volume mesh was built from the same surface mesh as the one used for the DSMC simulations (Figure 6). The objective was to use consistent surface meshes with the two solvers. Then, at the wall, prisms 


\begin{tabular}{ccccccc}
\hline \hline Run & Max. ref. level & $\lambda_{\infty}^{\mathrm{VHS}} / \Delta_{\mathrm{x}, \mathrm{y}, \mathrm{z}}$ & $\tau_{\text {coll }} / \Delta t$ & $N_{p}$ & $N_{c} / V_{\text {num }}\left(\cdot / \mathrm{m}^{3}\right)$ & $q_{\max }\left(\mathrm{kW} / \mathrm{m}^{2}\right)$ \\
\hline 1 & 3 & 1 & 1.6 & $1400 \times 10^{6}$ & $0.2 \times 10^{6}$ & 159 \\
2 & 4 & 1 & 2.1 & $1000 \times 10^{6}$ & $1.9 \times 10^{6}$ & 158 \\
3 & 3 & 2 & 2.1 & $900 \times 10^{6}$ & $3.9 \times 10^{6}$ & 156 \\
4 & 3 & 2 & 8.5 & $900 \times 10^{6}$ & $3.9 \times 10^{6}$ & 156 \\
\hline \hline
\end{tabular}

$N_{c} / V_{\text {num }}$ is the ratio between the number of cells and the domain volume.

TABLE 4: Refinement configuration parameters at $90 \mathrm{~km}$
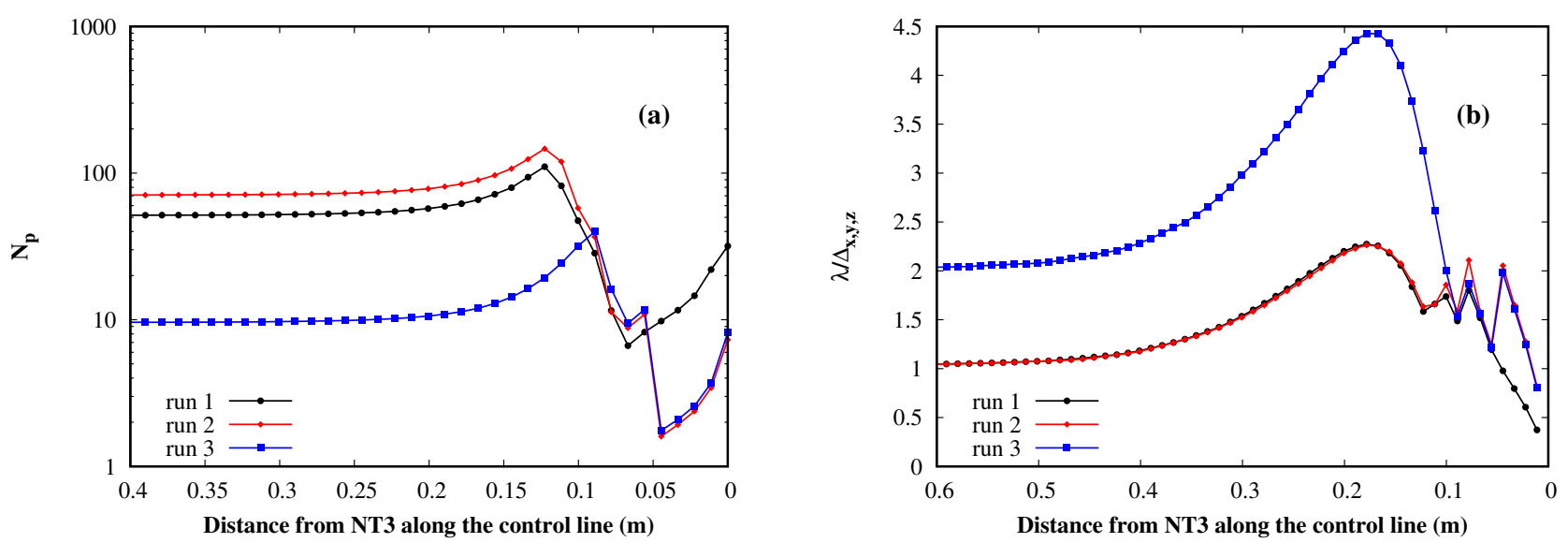

FiguRE 11: Comparison of the number of particles per cell (a) and of $\lambda_{\mathrm{x}, \mathrm{y}, \mathrm{z}} / \Delta_{\mathrm{x}, \mathrm{y}, \mathrm{z}}$ (b) along the control line for three refinement configurations at $90 \mathrm{~km}$.

with a first mesh size of $1 \mu \mathrm{m}$ and a height factor of 1.2 were built. A total of 50 prism layers were created all over the body except around the flaps where only 45 layers were extracted in order to avoid element overlapping. The shock interface envelope was extracted from the Mach contours of the DSMC solution and prism elements were extracted on both sides. Thus, in the vehicle direction, 15 layers were created with a minimum height of $0.5 \mathrm{~mm}$ and a height ratio of 1.2. In the opposite direction, 27 prisms layers of size between 0.5 and $2.15 \mathrm{~mm}$ were built with a 1.2 height ratio, leading to a total height of $50 \mathrm{~mm}$. Since the CFD shock was not predicted at the same location as that of the DSMC results, several meshes were done until the CFD shock was actually captured inside the dedicated prism layer. Finally, the remaining of the computational domain was filled with tetrahedral elements via a Delaunay procedure.

\subsubsection{Computational settings}

Our CFD simulations were performed with the ONERA code CEDRE [23] which is a multiphysics platform containing a large variety of solvers. For our study, the unstructured NavierStokes solver CHARME was used. In this work, the Navier-stokes equations were resolved with a second-order finite-volume discretization in space, with the flux vector splitting AUSM + scheme associated to a Van Leer limiter.

CHARME was designed as a mono-temperature solver assuming thermal equilibrium. The flow was assumed to be in chemical non-equilibrium and the Park's chemistry kinetics model with 5 species $\left(\mathrm{O}, \mathrm{N}, \mathrm{NO}, \mathrm{O}_{2}, \mathrm{~N}_{2}\right)$ and 17 reactions was used. By defaults, direct and inverse reactions are considered resulting in actually 34 reactions. However, the state of the gas (perfect or real, in chemical equilibrium or not) is not fixed by the user but is a consequence of the local flow conditions. In the same way, using a 5 species air model with 17 direct and inverse reactions does not necessarily mean that all species are present in the flow nor that all reactions occur. The influence of the chemical reactions are discussed in section 5. Furthermore, because of the low Reynolds number at $90 \mathrm{~km}\left(R e_{L_{\mathrm{ref}}, \infty}=12.6 \times 10^{3}\right)$ and the fact that the corresponding 


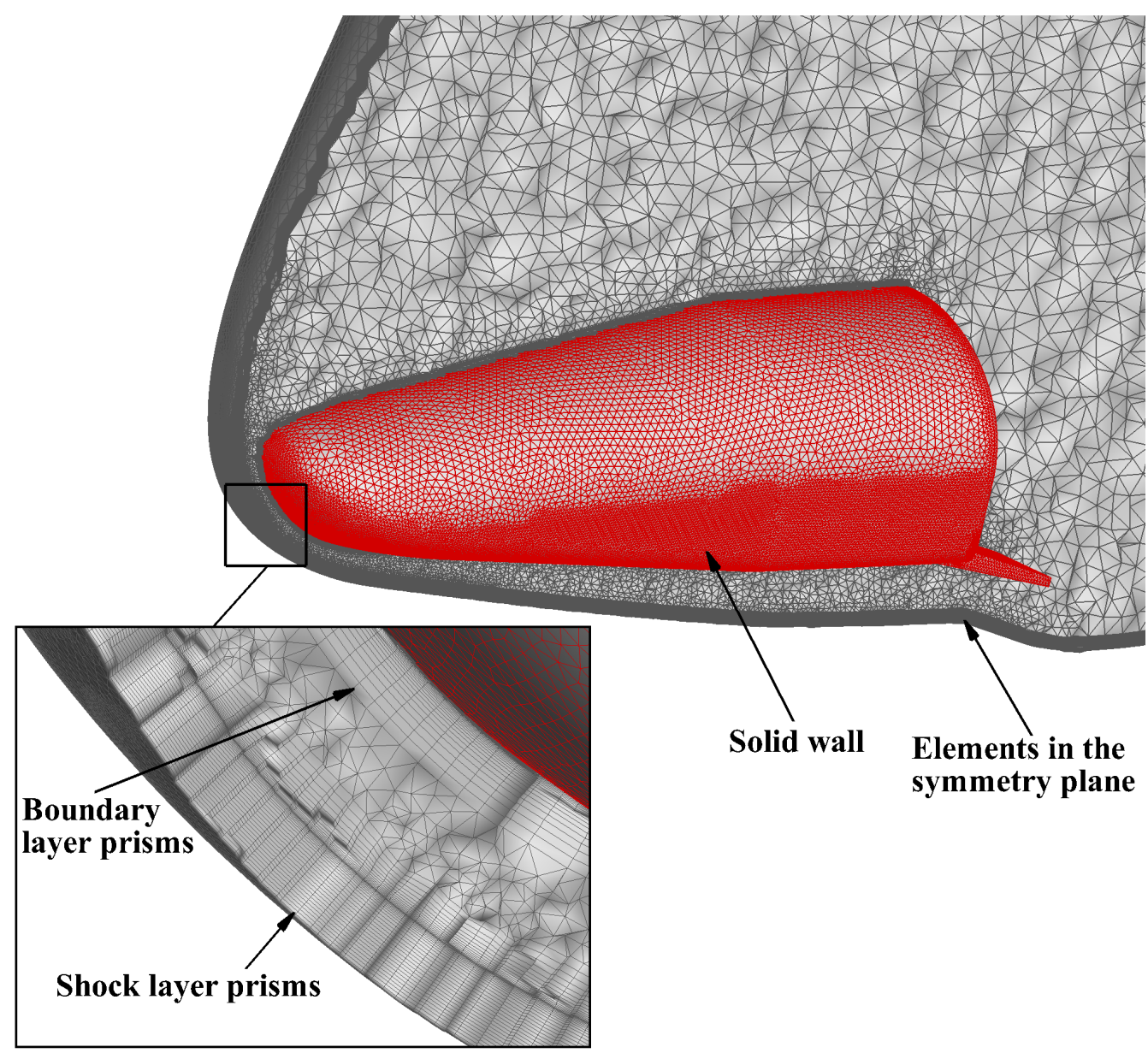

Figure 12: View of the 3D mesh and of the elements cutting the symmetry plane of the CFD computational domain.

DSMC simulation evidenced an attached flow, it was then assumed fully laminar and no particular refinement was deemed necessary behind the vehicle. Finally, a non-catalytic wall was supposed and the transport properties were computed as follows. The species conductivity was computed with a constant Prandtl number and the species viscosity was obtained from Blottner's model. The mixture viscosity and conductivity were computed through arithmetic averages of the species fraction.

\subsection{Continuum breakdown}

As evidenced by the Chapman-Enskog development [24, 20], the Navier-Stokes equations can be derived from a first order expansion of the Boltzmann equations in the Knudsen number. Hence, the Navier-Stokes equation hold only if small deviations from the Maxwellian equilibrium are assumed. In the transitional portion of atmospheric reentries, the characteristic length and the mean free path may significantly vary throughout the flowfield resulting in the simultaneous presence of continuum and rarefied regions. In this case, neither the application of a continuum or a rarefied method is fully satisfactory. The study of such flows resulted in the development of hybrid methods [25] which apply concurrently both methods in the appropriate regions of the flowfield. In this context, a continuum breakdown criteria indicates which method should be used in which region. When DSMC results are compared to the CFD results at the edge of their application spectrum, it is also common to use the continuum breakdown criteria to analyze the flowfield and to outline its rarefied regions.

Contrary to the global Knudsen number $K n$ based on the free stream mean free path $\lambda_{\infty}$ and 
the object's reference length $L_{\text {ref }}$ :

$$
K n=\frac{\lambda_{\infty}}{L_{\mathrm{ref}}}
$$

the continuum breakdown criteria consists in a local Knudsen number which can be defined in several ways [21]. A common Knudsen number is that of Boyd whose expression writes :

$$
K n_{\mathrm{GLL}}=\frac{\lambda}{Q}|\nabla Q|
$$

The flow property $Q$ can then either be taken as the temperature, the velocity or the density and $K n_{\mathrm{GLL}}>0.05$ usually indicates significant nonequilibrium [21]. The $K n_{\mathrm{GLL}}$ employed in this work is based on the density i.e. $Q \equiv \rho$.

\section{DSMC results analysis}

The main objective of this work is to assess the capacity of the DSMC method to properly simulate a complex vehicle reentry. Furthermore, a sensitivity analysis of some numerical parameters such as the accommodation coefficient, the chemical kinetic model or the collision model was conducted in order to identify the potentiel sources of discrepancy between the measurements and the numerical results. A particular attention was devoted to the comparison of the results obtained by DSMC and CFD in the near-continuum regime and in the light of the flight data.

\subsection{Analysis of the flowfield}

During an atmospheric entry, the flow goes from a free molecular regime to a continuum regime. Between these, several intermediary states are encountered and the nature of the flow changes radically. As incident and reflected particles begin to interact, the flow becomes transitional and a shock appears upstream the vehicle at a distance $\Delta s$ of the order of the mean free path $(\Delta s \sim \lambda)$. This first regime is called fully merged layer which means that the boundary layer and the shock layer are mixed in an indiscernible layer. This region is fully viscous and strong jump temperature and velocity slip effects appear. As the altitude decreases, the density rises exponentially and the flow enters the slip regime which includes the states of incipient merged layer and then of viscous layer introduced by Probstein in [26]. In this regime, the shock thickness $\delta s$ is of the order of the free stream mean free path $\lambda_{\infty}$ and the shock is distinguishable from the boundary layer. Progressively, the length of the temperature jump and velocity slip decreases $(\sim \lambda)$ and the shock layer is essentially inviscid. Finally, when the flow is in a fully continuum regime, the shock forms a discontinuity $(\delta s \ll \Delta s)$. The temperature jump and velocity slip effects vanish, the wall viscosity effects are negligible.

Hence, as the vehicle's altitude decreases, rarefaction effects weaken. The first consequence of this phenomenon is the reduction of the shock thickness, which transitions from a very weak thick shock to a stronger and thinner shock. The second consequence is the increase in the density and in the temperature across the shock as visible in Figure 13. The use of a gray-scale mapping similar to that of Banyai et al. [7] facilitates the comparison of our DSMC contours with theirs. The main difference being that for the lowest altitudes (lower than $105 \mathrm{~km}$ ), the 15 degree inclination of the flaps breaks the convex shape of the shock envelope.

Another high altitude interesting feature is the effect of the accommodation coefficient on the flowfield properties as well as on the surface quantities (pressure, friction, heat flux). The SPARTA code has been provided with a large variety of gas surface interaction models comprising among others, the standard Maxwell reflection model and the Cercignani Lampis Lord model. These two gas surface interaction models are the most used in the context of hypersonic rarefied flows. A detailed discussion of the gas surface interaction models [27] is out of the scope of this 
paper and a thorough investigation of their effects was not part of our objectives, which is why effects of accommodation were investigated with Maxwell model only.

Unlike for reentry vehicles, the study of gas-surface interaction for orbital aerodynamic applications is a highly active field of research. Thus, although the accommodation coefficient is rarely quantified for reentry materials, the study of satellite aerodynamic gives a good insight of the expected accommodation coefficient. Hence, Moe [28] and more recently Crisp et al. [29] addressed the issue of gas-surface interaction and evidenced that for Very Low Earth orbiting satellites (below $200 \mathrm{~km}$ ), the significant contamination due to adsorption results in accommodation coefficient values close to unity ( $w \approx 0.8$ to 1.0 ). Given the roughness of the IXV TPS and its likely contamination, the same order of magnitude would be expected. However, considering Moss and Bird's work on the Space Shuttle [30] in which values of accommodation coefficients down to 0.5 were considered as physically realistic, a similar study of influence was performed for the IXV.

The contours represented in Figure 14 illustrate the influence of the accommodation coefficient $(w=1$ and 0.5$)$ on the shock layer topology at $115 \mathrm{~km}$ of altitude. For such level of rarefaction, the particle interactions are dominated by the gas surface interactions. Indeed, when the mean free path is large enough, the particles reflected from the wall can travel large distances before interacting with other incoming particles. Hence, when a particle $i$ of total energy $\epsilon_{i}$ is specularly reflected from a wall, it can travel back to the shock layer while conserving a great amount of its energy that is then transferred to the neighboring particles. However, when the same particle $i$ is diffusely reflected from the wall, a part of its energy is directly transferred to the wall resulting in a energy $\epsilon_{i}^{*}$ smaller than $\epsilon_{i}$. Such particles provide less energy to the shock layer ultimately yielding to a thermally weaker shock. Finally, when the angle of attack is non null, particles can be reflected in a specific area of the shock which causes simultaneously a significant change in the shape of the density, the Mach number and the temperature contours (Figure 14). As the rarefaction level decreases with the altitude, the mean free path drops and the particles are trapped in the vicinity of the wall long enough for them to reach thermal equilibrium. The excess of

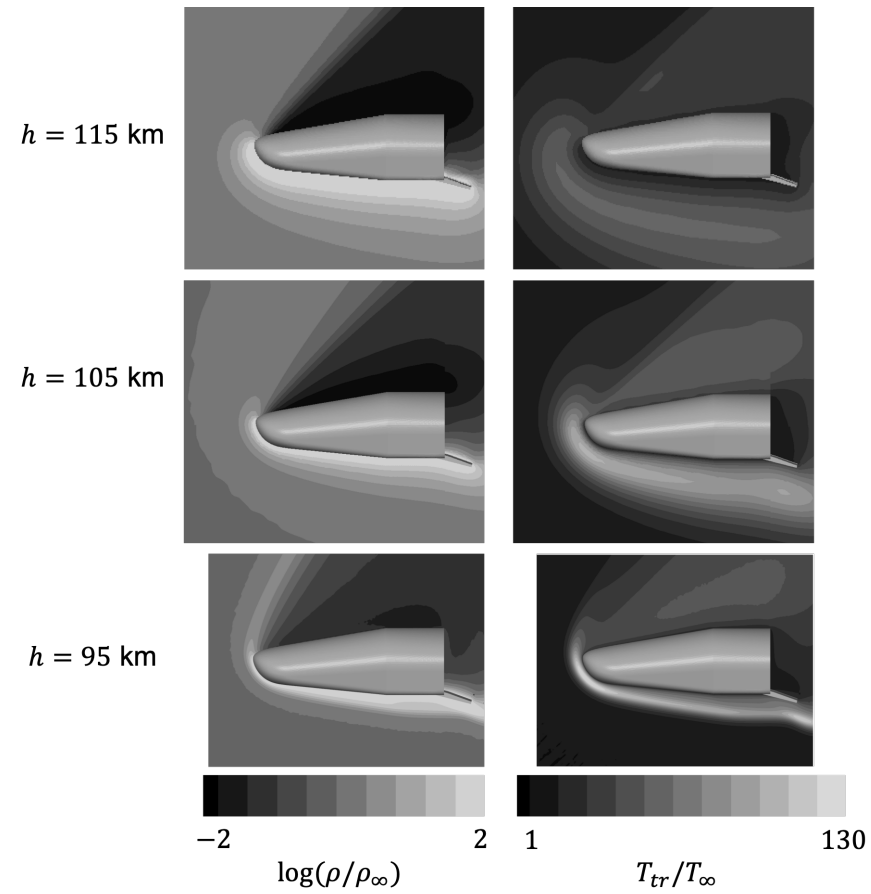

FIGURE 13: DSMC contours of normalized density (left) and translational temperature (right) between 115 and $95 \mathrm{~km}$.

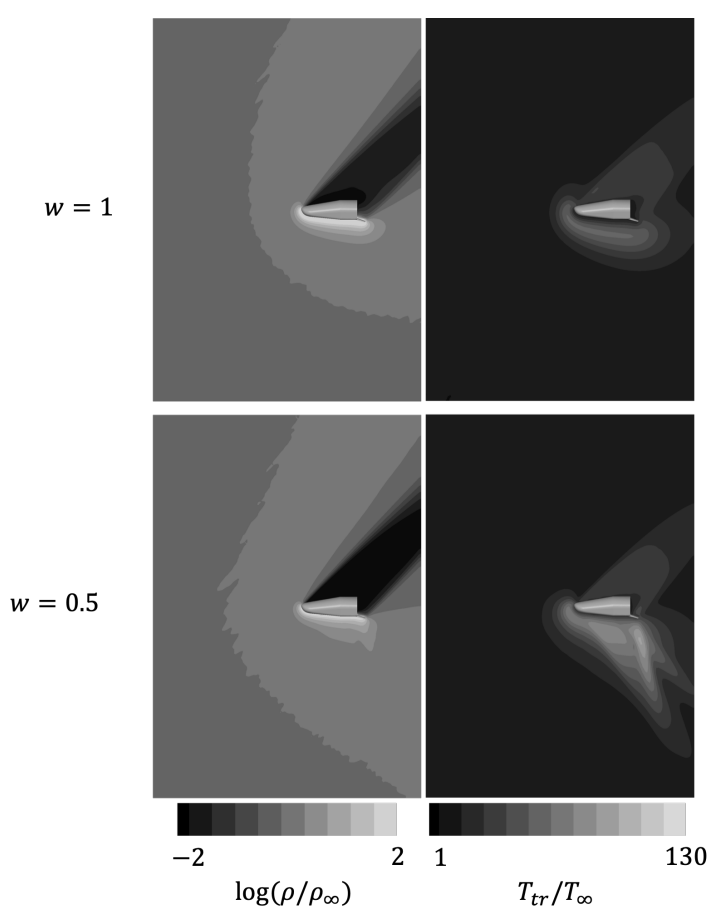

FIGURE 14: DSMC contours of normalized density (left) and translational temperature (right) for various accommodation coefficients $w$ at $115 \mathrm{~km}$. 
energy is dissipated near the wall and the shock layer is almost identical to that corresponding to fully diffuse conditions. At $100 \mathrm{~km}$ of altitude for instance, only the area surrounding the stagnation point is slightly more energized and this tendency progressively diminishes as the altitude decreases. In the shadow area, the density and the temperature are much smaller which is why the gas-surface interactions effects are less visible.

In DSMC, the transport quantities are not explicitly computed but are rather a consequence of the chosen collision model which is usually adjusted to reproduce experimental diffusion and viscosity data [19]. Consequently, one can wonder to what extend the choice of the collision model impacts the computed results.

As explained by Gimelshein and Wysong [31], the VHS intermolecular potential is one of the three major improvements of the DSMC method. This model offers several advantages such as the viscosity temperature dependence of an Inverse Power Law (IPL) model while using the Hard Sphere (HS) model scattering which guaranties an easy implementation and a high computational efficiency. For a given species, the VHS model is based on two experimentally determined coefficients : the reference species diameter $d_{\text {ref }}^{\mathrm{VHS}}$ and the temperature exponent $\omega^{\mathrm{VHS}}$ related to the viscosity $\mu$ by the relation :

$$
\mu \propto T^{\omega}
$$

As previously said in subsection 3.1.1, the Variable Hard Sphere (VHS) model configured with Bird's parameters was the standard collision model used for the computations presented herein. However, as indicated in [19], these parameters were obtained in specific experimental conditions. Indeed, the reference viscosity $\left(\mu_{\text {ref }}\right)$ was measured in standard atmospheric conditions $\left(T_{\text {ref }}=273 \mathrm{~K}\right.$ and $\left.P=101.325 \times 10^{3} \mathrm{~Pa}\right)$, the temperature exponent $\omega$ was inferred from viscosity measurements with $T \in[293 ; 373] \mathrm{K}$, and $d_{\text {ref }}$ was finally obtained from the relation between $\mu_{\text {ref }}$ and the VHS parameters. Since these temperature conditions are far from those considered herein, Bird's parameters can be deemed questionable. Moreover, the VHS model is not able to fit both the viscosity and the diffusion coefficients of the simulated gas. The Variable Soft Sphere (VSS) model [32] uses an additional experimentally based coefficient $\alpha^{\mathrm{VSS}}>1$ enabling to overcome the VHS limitation while having roughly the same numerical efficiency. Hence, for a specific species, one must specify a reference diameter $d_{\mathrm{ref}}^{\mathrm{VSS}}$, a temperature exponent $\omega^{\mathrm{VSS}}$ and the scattering parameter $\alpha^{\text {VSS }}$. In practice, there are two ways of implementing the collision models in a DSMC code. When the parameters are specified for each species, the parameters of a colliding pair of distinct species can be computed as an average of the pair species coefficients. In this case, one speak of collisionaveraged procedure which is that of SPARTA. Another procedure consists in specifying for each possible colliding pair of species the corresponding parameters. One then speak of collision-specific procedure which is that of MAP [33]. That procedure is more accurate and physically consistent for unlike species. Ways of determining pair specific coefficients are mentioned in [34t. According to [31], the VSS model should be preferred when reference diffusion data are available.

Hence, in order to assess separately the influence of using an optimized set of parameters and the effect of using the VSS model over the VHS model, several SPARTA computations were performed with the VHS and VSS models and the collision-averaged parameters derived by Stephani (Table 1 in [35]). These parameters $\left(d_{\text {ref }}, \omega\right.$ and $\alpha$ ) were fitted from realistic intermolecular potentials while assuming a constant reference temperature $T_{\text {ref }}=2880 \mathrm{~K}$ and by varying $\mathrm{T}$ between 1000 and $5000 \mathrm{~K}$. The temperature and velocity results obtained at $90 \mathrm{~km}$ are given in Figure 15.

According to the translational temperature (Figure 15a) and velocity (Figure 15b) profiles, the optimized VHS model slightly decreases the shock layer thickness. This is coherent with the fact that the collision model best-fit procedure described in [35] aims to improve the transport properties consistency between DSMC and CFD solvers. Indeed, by adjusting the collision model parameters to fit the transport properties computed with CFD approximations, the DSMC solver behavior should slightly move towards that of a CFD solver which has a tendency to predict 

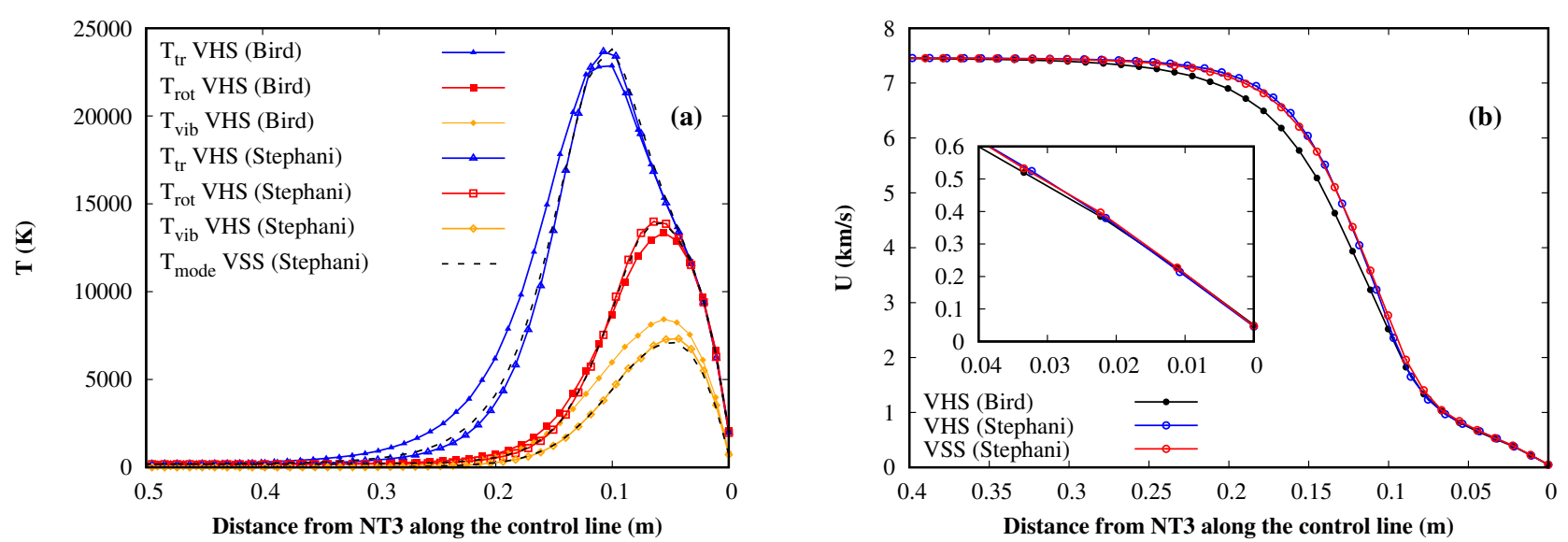

FIGURE 15: Influence of the collision model on the temperature (a) and velocity (b) profiles along the control line obtained with SPARTA at $90 \mathrm{~km}$.

thinner shocks. One denotes however that in these conditions, both optimized VHS and VSS models yield almost identical results.

The next subsections focus on the surface quantities. They comprise the analysis of the computed aerodynamic results and the comparison of the numerical heat flux with the collected flight data.

\subsection{Aerodynamic results analysis}

As previously discussed in introduction, Banyai et al. [7] have investigated in details the aerodynamic of the IXV between 120 and $85 \mathrm{~km}$, in preparation of the vechicle's mission. In this part, the objective is to analyze the effective aerodynamic performance of the vehicle thanks to the precise knowledge of its flight conditions.

The vehicle coordinate system is defined in Figure 16. Figure 17 shows the evolution of the aerodynamic coefficients with the altitude and Figure 18 gives the evolution of the pressure and friction coefficients distributions along the centerline at 115, 100 and $90 \mathrm{~km}$.

The first consequence of the air densification is the rapid increase of the surface pressure which more than doubles every $5 \mathrm{~km}$. The pressure coefficient progressively decreases and the stagnation value falls within the range of values predicted by the analytic expression based on the Pitot Rayleigh formula $\left(C_{p \text {,stag }}=1.84\right.$ for $\gamma=1.4$ and $C_{p \text {,stag }}=1.9$ for $\left.\gamma=1.1\right)$. In the meantime, the skin friction coefficient distribution $C_{f}$ drops from a mean value of 0.7 at $115 \mathrm{~km}$ down to 0.1 at $90 \mathrm{~km}$ (Figure 18). As explained in section 4.1, the wall friction diminution comes from the diminution of the viscous effects approaching the continuum regime.

In terms of aerodynamic forces and moments (Figure 17), the pressure increase induces an increase in the moment coefficient $\left(C_{\mathrm{m}, \mathrm{cg}}\right)$, and a diminution of the normal $\left(C_{N}\right)$ and axial $\left(C_{A}\right)$ force coefficients. As indicated by Banyai et al. in [7], the small values of $C_{\mathrm{m}, \mathrm{cg}}$ (Figure 17b) show that the vehicle is stable during the transitional portion of the reentry and the progressive increase toward a null value as it approaches the continuum regime ensures a stable transition towards the denser layers of the atmosphere. Finally, as envisioned by Banyai et al., for the altitudes greater than $100 \mathrm{~km}$, the moment resulting from the aerodynamic forces might be incapable of counteracting the vehicle's possible inertia due to a disturbance. Nevertheless, the stability shown by the trajectory data and the nominal behavior of the vehicle indicate that such perturbations did not occur.

With respect to the accommodation coefficient, Figure 17 shows that decreasing the accommodation coefficient leads to lower values of $C_{A}$ and to higher values of the aerodynamic coefficients $C_{N}$ and $C_{\mathrm{m}, \mathrm{cg}}$. As explained by Padilla in [27], a decrease of the accommodation coefficient induces an increase in the gas energy around the vehicle that benefits the pressure applied to its wall. 
This behavior is illustrated in Figure 18a where a significant increase in the surface pressure is observed at $115 \mathrm{~km}$ as the accommodation coefficient is lowered. The friction coefficient behavior is the opposite (Figure 18b). In accordance with the previous observations, Figures 16 and 18 show that the sensitivity to the accommodation coefficient decreases sharply as the altitude diminishes.

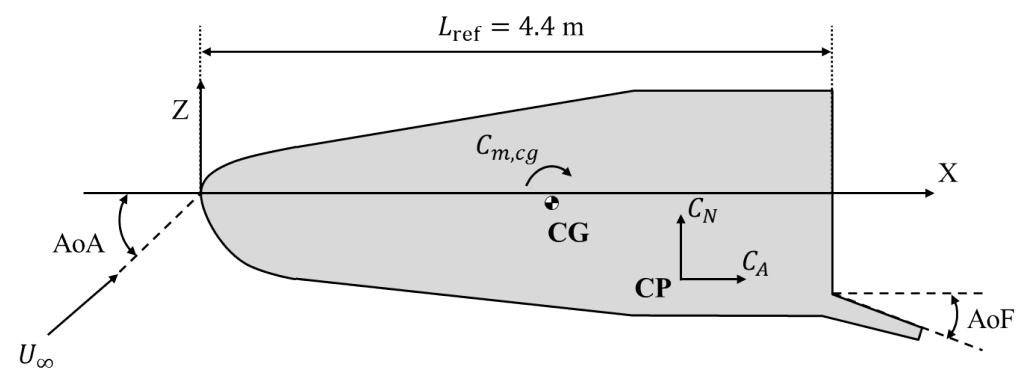

FIGURE 16: IXV coordinate system with the center of gravity CG located at $(x, y, z)=(2.58,0,-0.08) \mathrm{m}$.
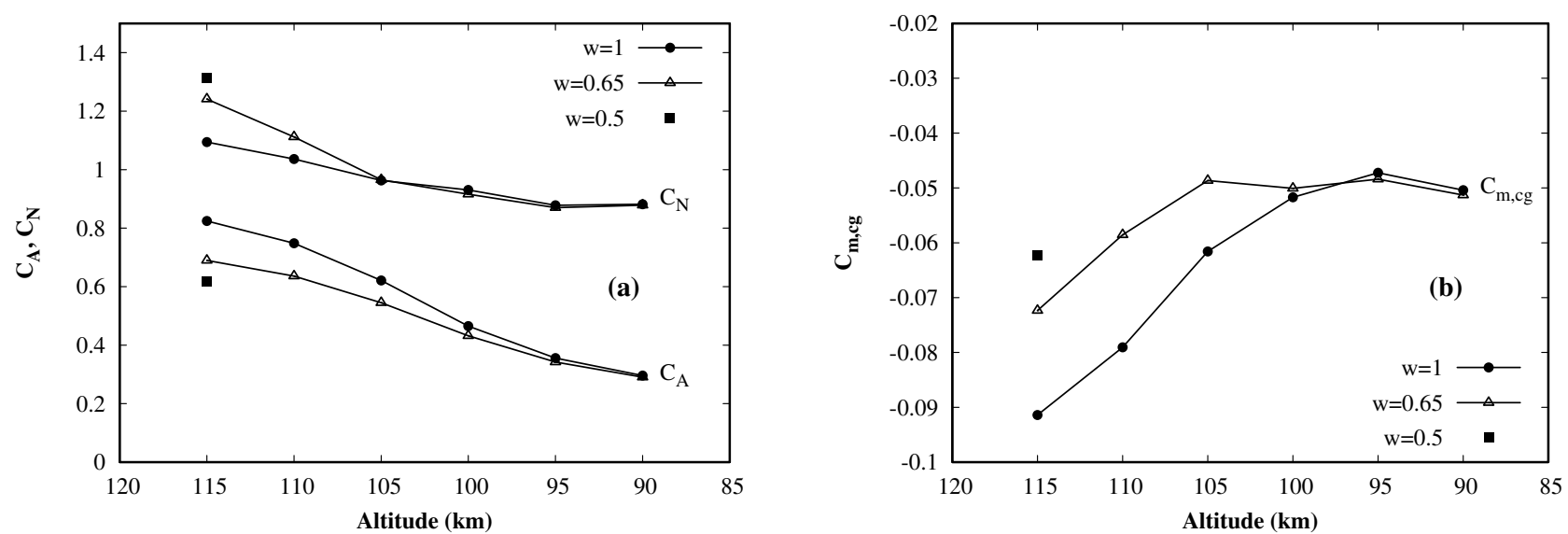

FIGURE 17: Evolution of the normal and axial force coefficients (a) and pitching moment coefficient (b) obtained with SPARTA between 115 and $90 \mathrm{~km}$ (note that the results for $w=0.5$ is given for the $115 \mathrm{~km}$ point only).

In DSMC, the surface pressure related quantities are derived from the momentum change of the impinging particles. Hence, when a particle $i$ impacts a wall element of surface $\delta s$, the momentum change $\delta \overrightarrow{p_{i}}$ writes :

$$
\delta \overrightarrow{p_{i}}=m_{i}\left(\vec{v}_{i}^{\prime}-\overrightarrow{v_{i}}\right)
$$

where $m_{i}$ is the particle mass and $\vec{v}_{i}^{\prime}$ and $\overrightarrow{v_{i}}$ respectively denote the post and pre-collision velocities of the particle. For each time step $\Delta t$, the pressure components are then derived from the cumulative momentum change occurring in the given time step. Considering the normal pressure $P_{s}$ applied to an element $\delta s$ in a given time step for instance, its value is computed as :

$$
P_{s}=-\sum_{i}^{n_{t}} \frac{\delta \overrightarrow{p_{i}} \cdot \overrightarrow{n_{s}}}{\delta s \Delta t f_{\text {num }}},
$$

with $n_{t}$ the number of impinging particles during the time step, $\overrightarrow{n_{s}}$ the surface element normal and $f_{\text {num }}$ the ratio of physical particles to the numerical particles.

The relation (5) shows that since the momentum change is proportional to the particles pre and post-collision differences, the resulting pressure components are weakly affected by the collision model, the chemical composition and by the internal energy modes. However, since the surface pressure is proportional to the free stream density, a significant uncertainty on the density results yields a roughly similar uncertainty on the aerodynamic coefficients (see Figure 19). 


\subsection{Aerothermodynamic results analysis}

\subsubsection{General analysis}

The flight data and the DSMC heat flux along the lines represented in Figure 5 are given in Figures 20, 21, 22 and 23 for various flight points. In compliance with TASI's demand, the Figures including flight data are represented in a non-dimensional form (all values are divided by the same reference heat flux $\left.q_{\mathrm{ref}}\right)$. However, among the 20 thermocouples considered here, the heat flux of 11 of them are available in Appendix E.3 (pp 268-271) of [6].

First focusing on the flight data themselves, Figure 20 shows that between 115 and $100 \mathrm{~km}$, the thermocouples WT62, WT48 and WT12 located on the windward seem to slowly recover.
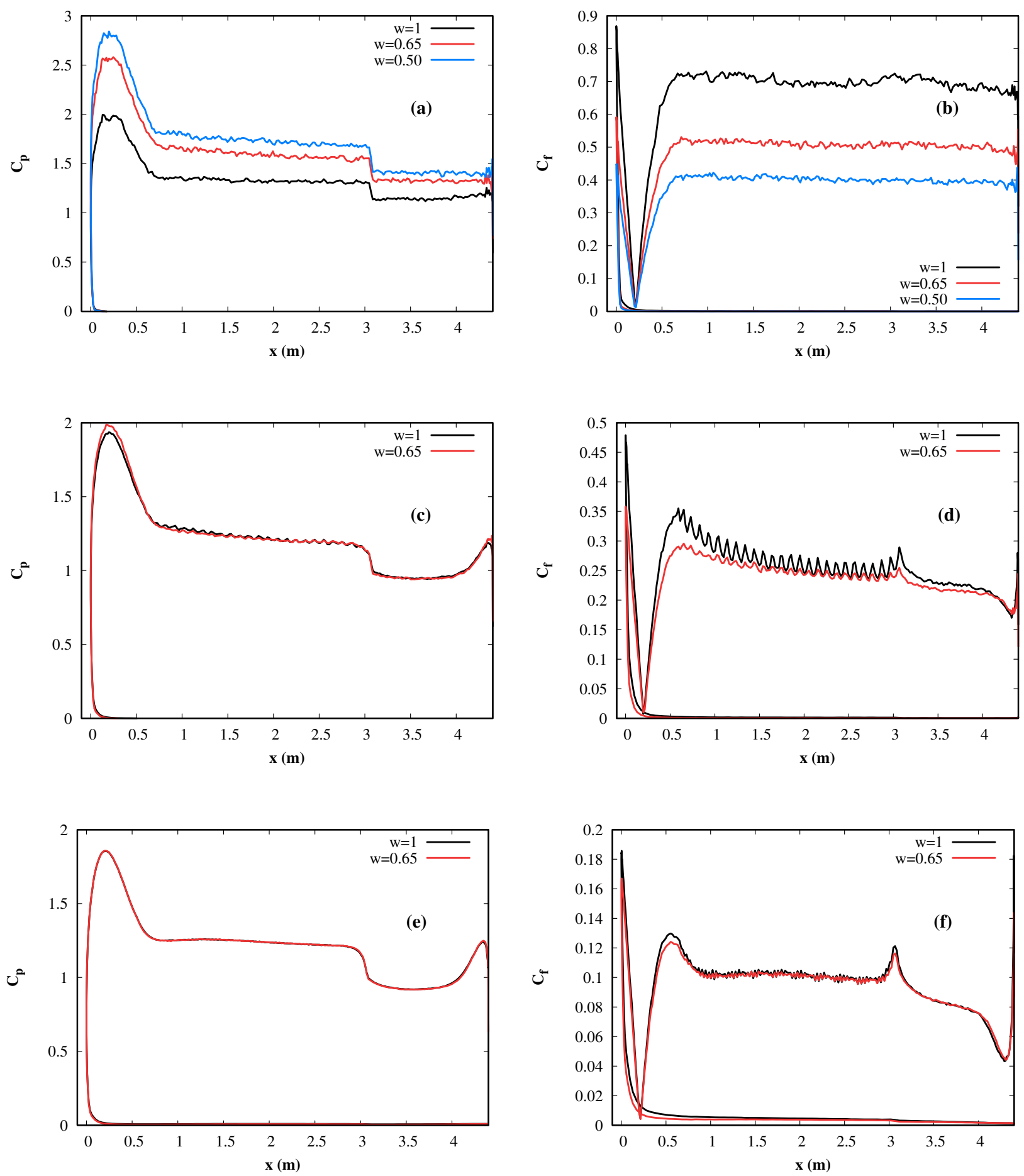

FIgURE 18: Influence of the accommodation coefficient on the DSMC pressure (left) and skin friction (right) coefficient distributions along the vehicle windward centerline at 115 (a-b), 100 (c-d) and 90 (e-f) km. 

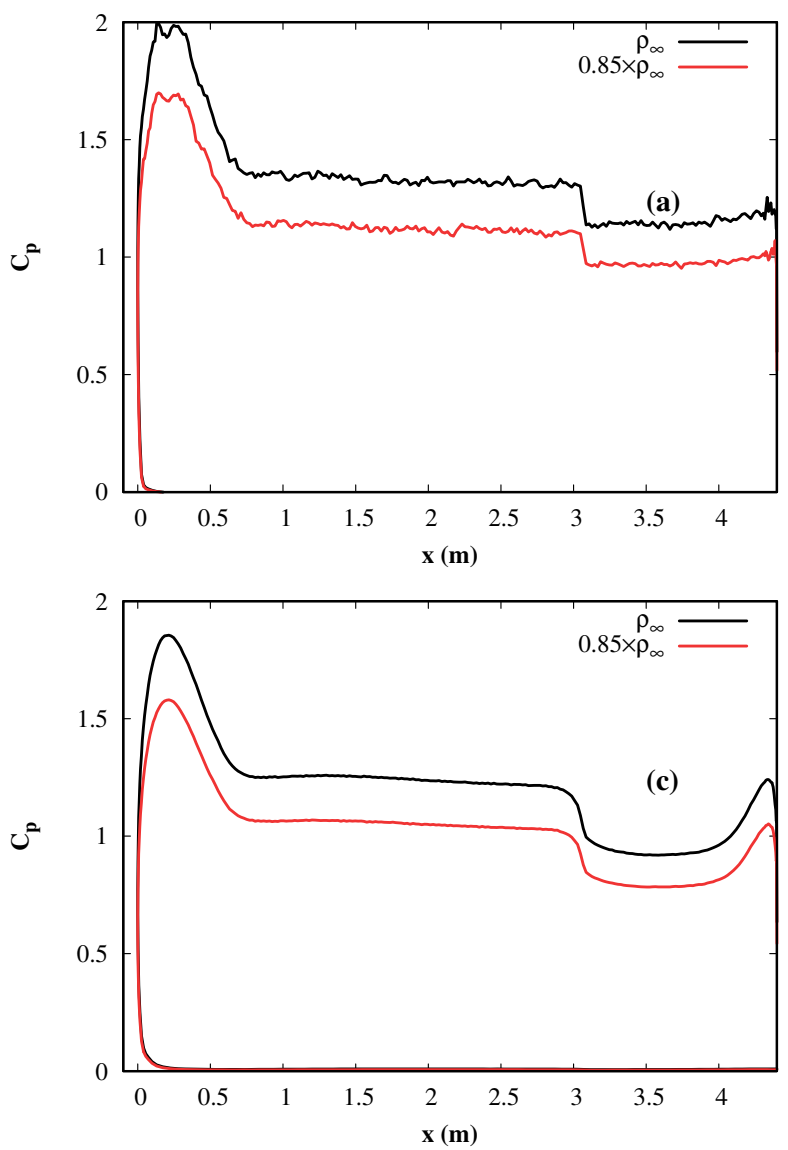
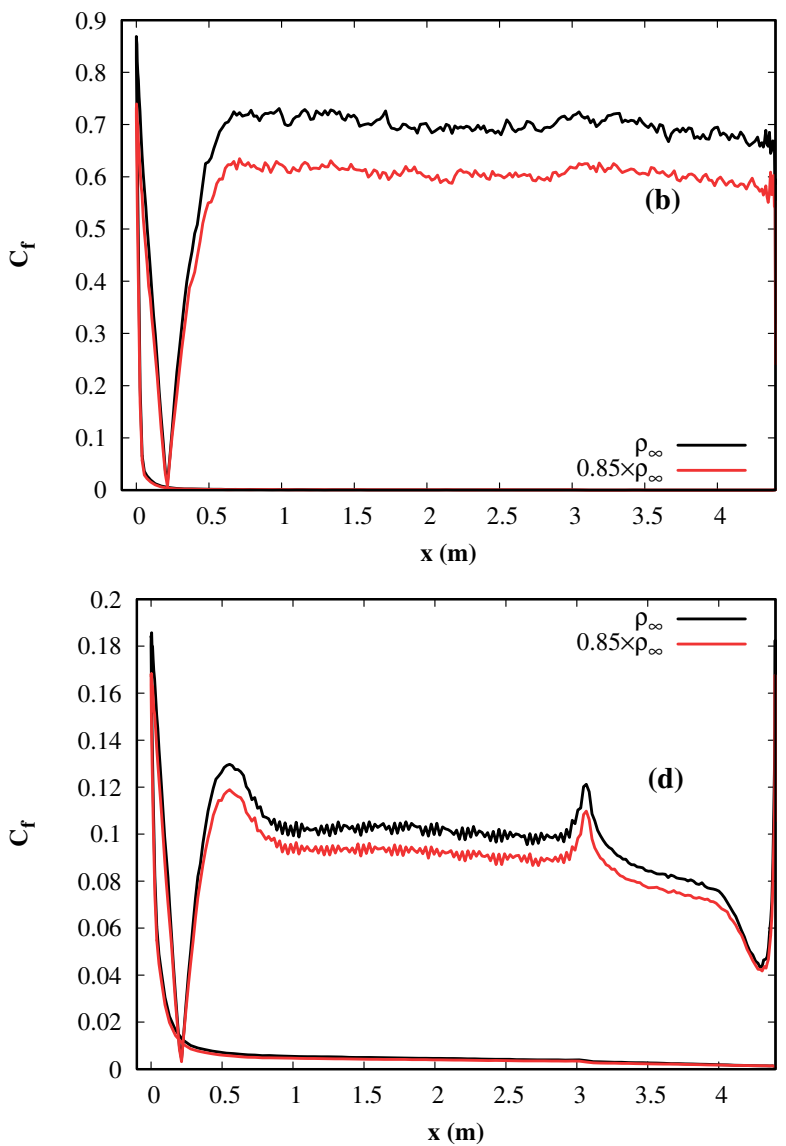

FIGURE 19: Influence of a $15 \%$ free stream density uncertainty on the DSMC pressure (left) and skin friction (right) coefficients distributions along the vehicle windward centerline at 115 (a-b) and 90 (c-d) km.

Indeed, for these TC, the reconstructed heat flux are abnormally low down to $110 \mathrm{~km}$. For the lower altitudes, these reconstructed heat flux line up with the values obtained at the neighboring sensors locations. This tendency could be due to the fact that for the highest altitudes, the wall temperature value in the windward area is too close to the thermocouple sensitivity. For all altitudes, the reported values for the thermocouples WT12 and NT2 are not coherent with the neighboring thermocouples values which indicate probable malfunction.

In Figures 21, 22 and 23, the same tendency is observed. For the altitudes between 115 and 105 $\mathrm{km}$, the experimental heat flux follow the expected shape. However, for the altitudes below 105 $\mathrm{km}$, the thermocouples WT41, WT66 and WT80 located in the shoulder region $\left(\theta \in\left[30^{\circ} ; 50^{\circ}\right]\right.$ in Figure 21 to 23) capture the heat flux peak but with a substantially lower magnitude than that expected in this kind of trailing edge region.

For all altitudes, the standard DSMC results (i.e. with $w=1$ ) are much higher than the flight data. With respect to the measurements, Table 5 summarizes the relative differences visible in Figure 20. The surprisingly large differences for the 100 and $95 \mathrm{~km}$ altitudes compared to that of the neighboring flight points suggest that anomalies in the atmospheric conditions might have occurred for these altitudes.

Furthermore, the results show that, as expected, the atmosphere densification induces a significant increase of the convective heat flux and a decrease in its sensitivity to the accommodation coefficient. Indeed, between 115 and $105 \mathrm{~km}$, the influence of the accommodation coefficient on the numerical heat flux is significant enough to reach a good agreement with the flight data in the nose region of the vehicle. However, this aspect is not entirely satisfactory since different values of the accommodation coefficient are required and permit to match some of the flight data only.

In order to evaluate the possible sources of discrepancies between the numerical results and 

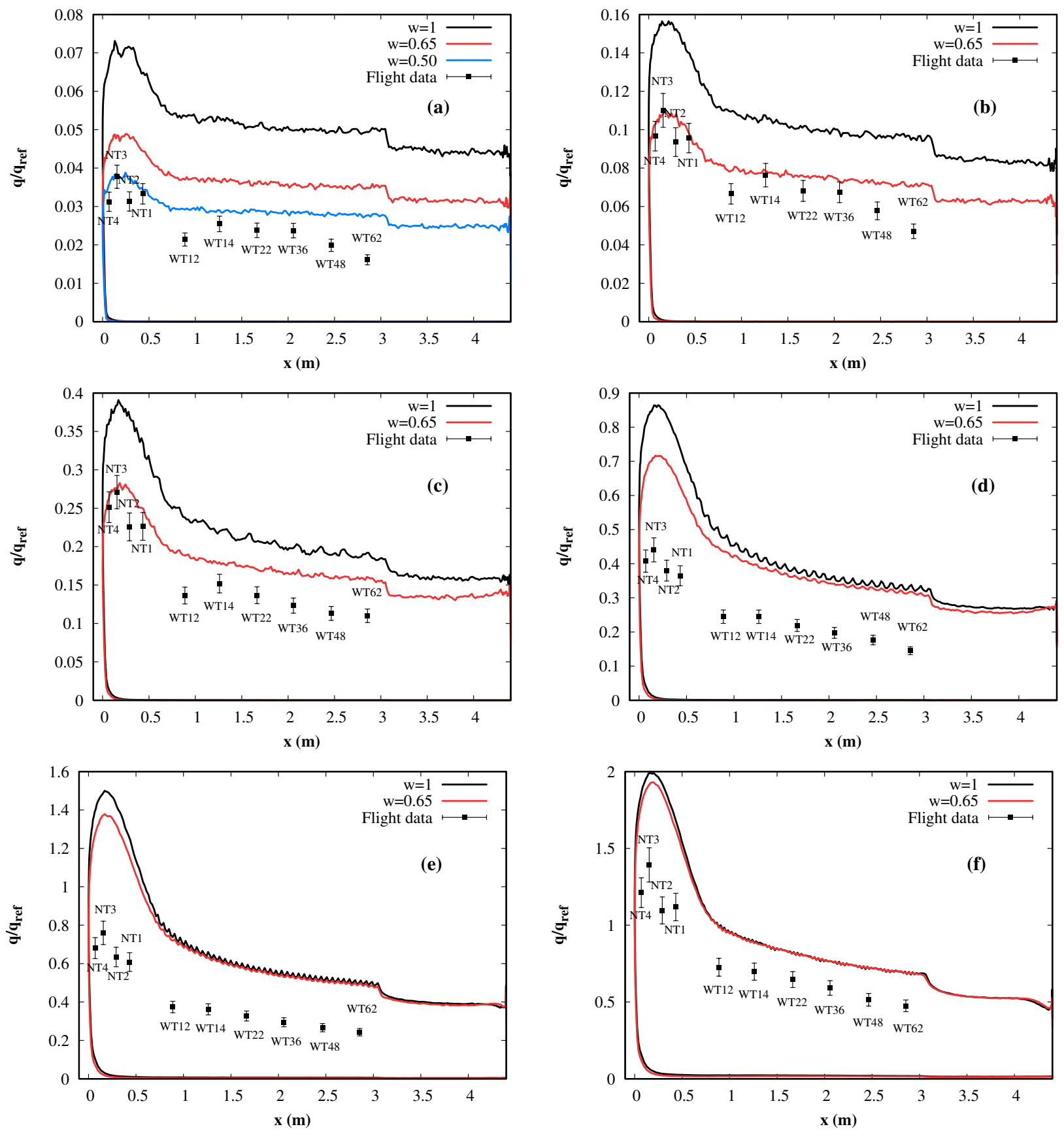

Figure 20: Comparison of the flight data and the DSMC heat flux distribution along the vehicle windward centerline obtained with various accommodation coefficients at 115 (a), 110 (b), 105 (c), 100 (d), 95 (e) and 90 (f) $\mathrm{km}$.

the flight data, a sensitivity study to various numerical parameters was performed. These include the accommodation coefficient, the model of chemistry and the chemical kinetics, the model of collision and the uncertainty to free stream conditions.

\subsubsection{Sensitivity analysis to the accommodation coefficient}

As discussed in the previous section, specular reflections deprive the wall from a certain amount of the impinging particles energy. When the rarefaction level of the flow is significant enough, this yields a significantly smaller heat flux, roughly proportional to the accommodation coefficient ${ }^{2}$

2. The expression of the heat flux in the free molecular regime derived from the kinetic theory yields proportionality between the heat flux and the accommodation coefficient [20]. 

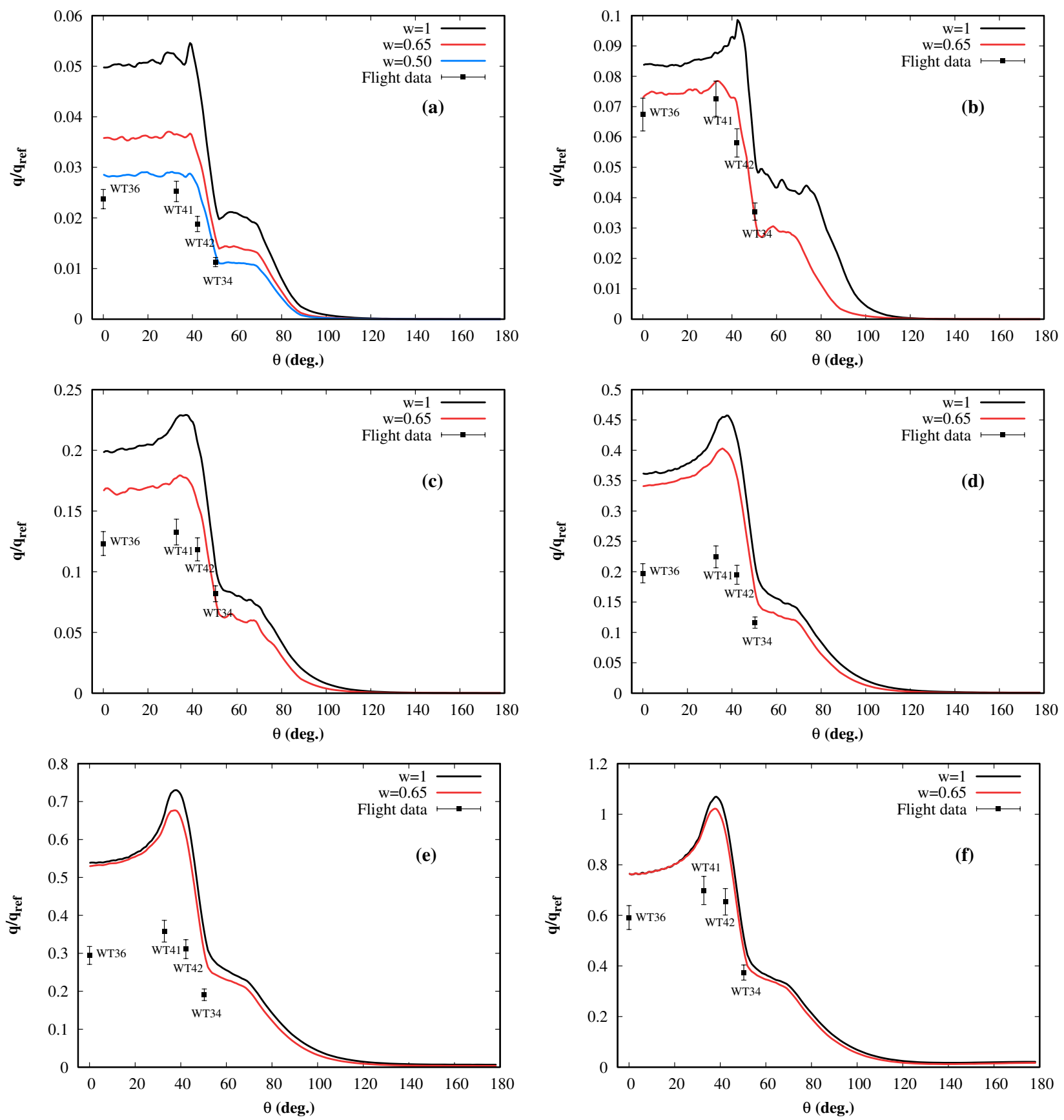

FIGURE 21: Comparison of the flight data and the DSMC heat flux distribution along the $x=2.06 \mathrm{~m}$ line obtained with various accommodation coefficients at 115 (a), 110 (b), 105 (c), 100 (d), 95 (e), 90 (f) km.

(see Figures 20a-20c). This is due to the fact that when the accommodation coefficient is smaller, less thermal energy is transferred to the wall in order to accommodate the gas thermal energy with that of the wall [27]. As the altitude decreases, here below $100 \mathrm{~km}$, the accommodation coefficient influence gets smaller and smaller until its effect only becomes discernible in the nose region of the vehicle. This phenomenon can be explained by the particles mean motion which follows the streamlines driving them away from the nose before wall thermal equilibrium can be reached. Yet, since the particles remain close to the wall, the stream which contains those specularly reflected particles travels along the vehicle's wall and blends with those coming from the free stream. If thermal equilibrium is not already reached in the wall vicinity, the particles that were previously specularly reflected keep colliding with the wall sometimes transferring an extraamount of energy, superior to that of the particles coming from the free stream and compensating the part of specularly reflected particles. Ultimately, this can result in identical heat flux down 

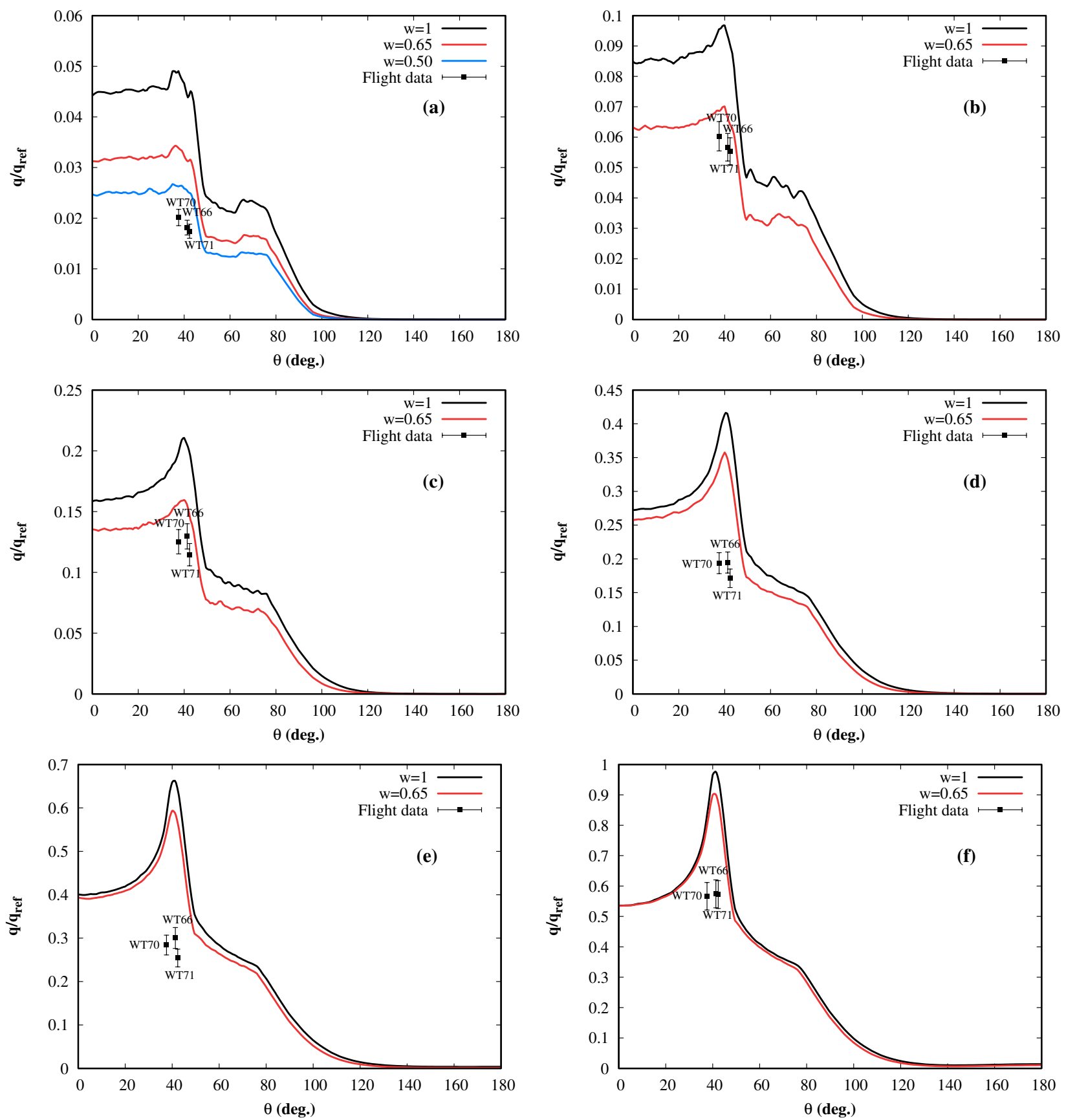

Figure 22: Comparison of the flight data and the DSMC heat flux distribution along the $x=3.52 \mathrm{~m}$ line obtained with various accommodation coefficients at 115 (a), 110 (b), 105 (c), 100 (d), 95 (e), 90 (f) km.

the nose (see Figures 20d to 20f) and away from the shoulder (i.e. for $\theta<40^{\circ}$ and $\theta>50^{\circ}$ - see Figures 21d to 21f, 22d to 22f, 23d to 23f).

Finally, given the similarities between the STS and the IXV flights, these results can be analyzed concurrently with Moss and Bird's DSMC work [30] whose results were unable to match the STS-2 heat flux measurements at $110 \mathrm{~km}$ of altitude even with an accommodation coefficient of 0.5 . This inability could be due to an approximate knowledge of the free stream density $(60 \%$ greater than ours at $110 \mathrm{~km}$ ) which probably prevented them from retrieving the measurements even with a small accommodation coefficient. However, as discussed in Section 4.1, the use of a non diffuse boundary condition is a priori not justified and the low value of the STS measurements at $110 \mathrm{~km}$ could be due, like in the IXV case, to an inappropriate instrumentation.

In DSMC, the wall heat flux is computed from the post and pre-collision energy difference of 

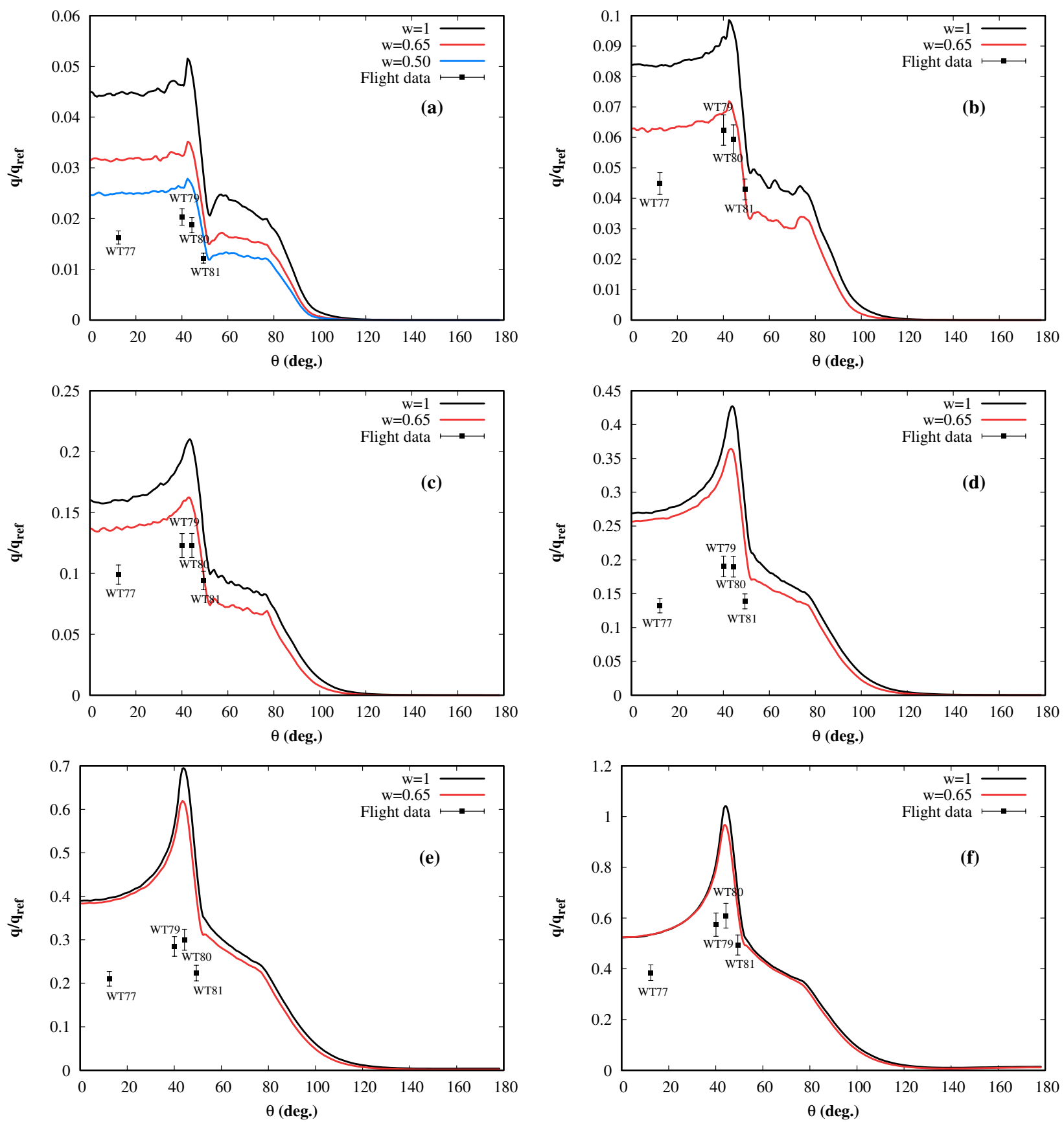

Figure 23: Comparison of the flight data and the DSMC heat flux distribution along the $x=3.99 \mathrm{~m}$ line obtained with various accommodation coefficients at 115 (a), 110 (b), 105 (c), 100 (d), 95 (e), 90 (f) km.

the impinging particles. Hence, a particle $i$ interacting with the wall would lose the kinetic energy $\delta \epsilon_{k, i}$ written as :

$$
\delta \epsilon_{k, i}=\frac{1}{2} m_{i}\left(v_{i}^{\prime 2}-v_{i}^{2}\right) .
$$

One must also consider the loss of energy in each internal energy mode $\delta \epsilon_{\text {mode }, i}$ :

$$
\delta \epsilon_{\text {mode }, i}= \begin{cases}\epsilon_{\text {mode }, i}^{\prime}-\epsilon_{\text {mode }, i} & \text { if } i \text { is polyatomic } \\ 0 & \text { otherwise }\end{cases}
$$

where $\epsilon_{\text {mode }, i}^{\prime}$ and $\epsilon_{\text {mode }, i}$ respectively denote the post and pre-collision energy of a given internal mode of energy. Finally, the total heat flux $q_{s}$ that is transmitted to a surface element $\delta s$ in a 
given time step writes :

$$
q_{s}=-\sum_{i}^{n_{t}} \frac{\delta \epsilon_{k, i}+\delta \epsilon_{\mathrm{rot}, i}+\delta \epsilon_{\mathrm{vib}, i}}{\delta s \Delta t f_{\mathrm{num}}} .
$$

This time, equation (7) shows that the heat flux is not function of a simple pre/post-collision velocity difference (see equation (5)) but rather a second order difference. As a consequence, the dependency of the heat flux is expected to be greater to some of the factors previously mentioned (intermolecular collision model and chemical composition).

\subsubsection{Sensitivity analysis to the chemical reaction model}

The DSMC method provides several models for the simulation of chemical reactions. Although $a b$ initio based reacting models are under development [22] and have recently proved to be usable for complex 5-species air reacting flows [36], this kind of model is currently not implemented in most DSMC codes. The prevalent models are Bird's Total Collision Energy (TCE) [19] and Quantum-Kinetic (Q-K) [37, 38] models. The main advantage of the latter being that it takes into consideration the vibrationally favored aspect of the dissociation reactions [31]. According to Bird [39], the Q-K and TCE models give similarly good results when they are used with reliable data. Otherwise, the Q-K model is expected to supersede its homolog. In SPARTA, the Q-K model is implemented [40, 41] and Figure 24 shows the difference between both models using Park rate coefficients at $90 \mathrm{~km}$ and in the standard conditions.

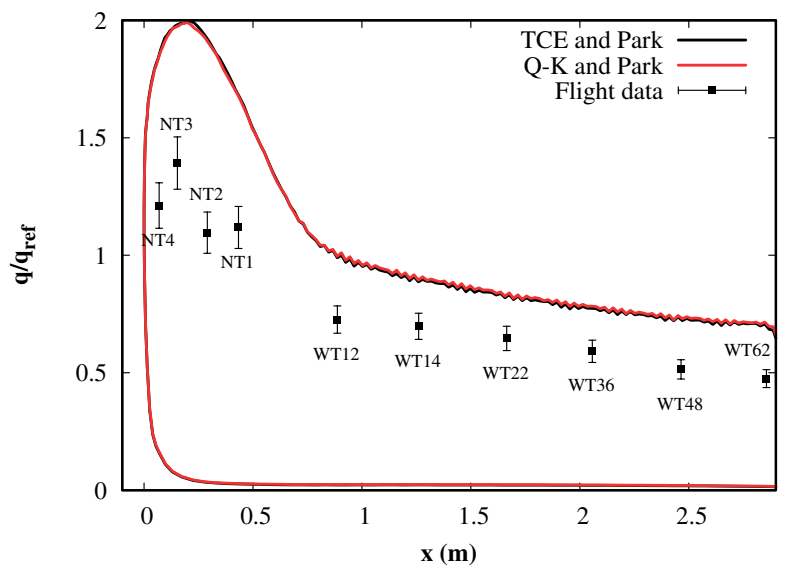

Figure 24: Comparison of the flight data and the DSMC heat flux along the vehicle windward centerline obtained with two chemical models at $90 \mathrm{~km}$.

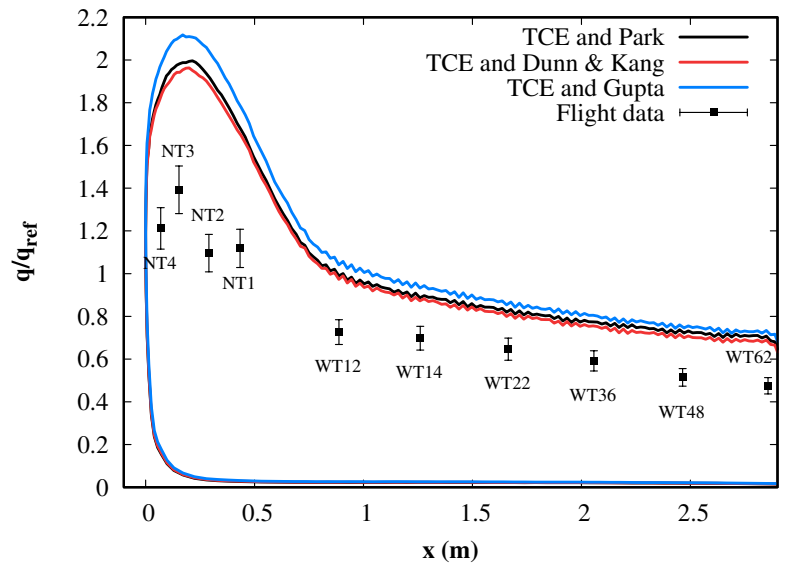

Figure 25: Comparison of the flight data and the DSMC heat flux along the vehicle windward centerline obtained with three chemical kinetic models at 90 $\mathrm{km}$.

\begin{tabular}{l|ccccc}
\hline \hline & \multicolumn{5}{|c}{$\left(q_{\text {num }}-q_{\exp }\right) / q_{\exp }(\%)$} \\
Altitude $(\mathrm{km})$ & WT62 & WT36 & WT14 & NT1 & NT3 \\
\hline 115 & 207 & 109 & 109 & 95 & 92 \\
110 & 104 & 47 & 40 & 46 & 43 \\
105 & 75 & 60 & 45 & 47 & 42 \\
100 & 130 & 86 & 71 & 106 & 97 \\
95 & 105 & 83 & 83 & 104 & 94 \\
90 & 48 & 29 & 26 & 50 & 43 \\
\hline \hline
\end{tabular}

TABLE 5: Summary of the relative difference between the DSMC and the experimental heat flux at various locations along the centerline. 
Although SPARTA log files indicate a significantly different number of each kind of reaction occurrence, no significant difference is visible in the predicted heat flux. Considering the flowfield temperature $\left(T_{\mathrm{tr}}<25000 \mathrm{~K}\right)$, this result supports Bird's comment in [39] and confirms the accuracy of the TCE model 40 years after its appearance [31].

In addition to its simplicity and numerical efficiency, the TCE model reaction cross-section (i.e. probability of reaction) is directly related to the simulated reaction rates. Hence, this model is capable of capturing any measured equilibrium reaction rate expressed in Arrhenius form while being applicable to nonequilibrium flows [31]. However, since multiple sets of chemical kinetic rate coefficients exist, the TCE model can provide as many solutions as there are chemical kinetic models. A priori, the behavior of a given global chemical kinetic model can be described as more or less rapid than another. Indeed, the more rapid the chemical kinetics, the more it is supposed to dissociate and recombine. However, the recombination reactions require more energy than the dissociation reactions which is why they generally do not get activated in the very low pressure conditions of the rarefied regime. Since dissociation reactions are endothermic, they consume a portion of the flow energy which ultimately yield a smaller heat flux ${ }^{3}$. On the contrary, when the overall chemical kinetics is slower, the heat flux is expected to be greater. In practice, endothermic reactions are in competition with exothermic reactions (i.e. recombination and reverse exchange reactions) and the different chemical kinetic models are not that easily classifiable. Indeed, in [43], Wang et al. investigated the performance of 4 chemical kinetic models (Dunn \& Kang, Park 1987, Park 1991 and Gupta) for heat transfer applied to three hypersonic vehicles (ELECTRE, Apollo and the Space Shuttle Orbiter). Their results show that for the tested vehicles, the chemical kinetic models produced heat flux in good agreement with the available flight data and with each others for simple geometries (i.e. ELECTRE and the Space Shuttle centerline). For the more complex regions (i.e. Apollo windside shoulder and the Space Shuttle wing leading edge) the chemical kinetic models evidenced greater discrepancies in the computed heat flux. Another of their conclusions is that to this day, the chemical kinetics are complex and the related phenomena are not well understood [43].

The DSMC heat flux obtained with Park, Gupta and Dunn \& Kang chemical kinetic models is given in Figure 25. The influence of the chemical kinetic models is mostly located around the nose of the vehicle. Indeed, it is the region where the flow is thermally the most severe and thus the region where the differences in the reaction rates have the major impact on chemical composition and heat flux. A very good agreement is observed between Park and Dunn \& Kang models since the heat flux obtained with Dunn \& Kang coefficients is less than $2 \%$ smaller in the nose region than that obtained with Park's coefficients. With Gupta's model, the difference is more important and an increase of around $6 \%$ is obtained in this region with respect to the results obtained with Park's model.

In conclusion, the comparison of the chemical kinetic models evidenced moderate differences in the IXV nose region and negligible discrepancies elsewhere. This can be explained by the fact that since the thermal conditions are the most severe in the nose region, the chemical phenomena are more significant and the differences are more pronounced. Nevertheless, a specific choice of chemical or chemical kinetic model does not provide an explanation for the deviations between the numerical results and the flight data.

\subsubsection{Sensitivity anlysis to the collision model}

As previously discussed, the collision models configured with Stephani's parameters [35] have proved to slightly modify the flowfield around the vehicle at $90 \mathrm{~km}$. Moreover in [30], Moss and Bird already underlined the importance of the collision model for the numerical reconstruction of heat flux data. Their paper even indicates that an adjustment of the VHS collision model parameters could yield a change up to $10 \%$ for their heat flux computations.

3. This observation is easily visible for instance when the same flow is considered as reacting or not [42]. 

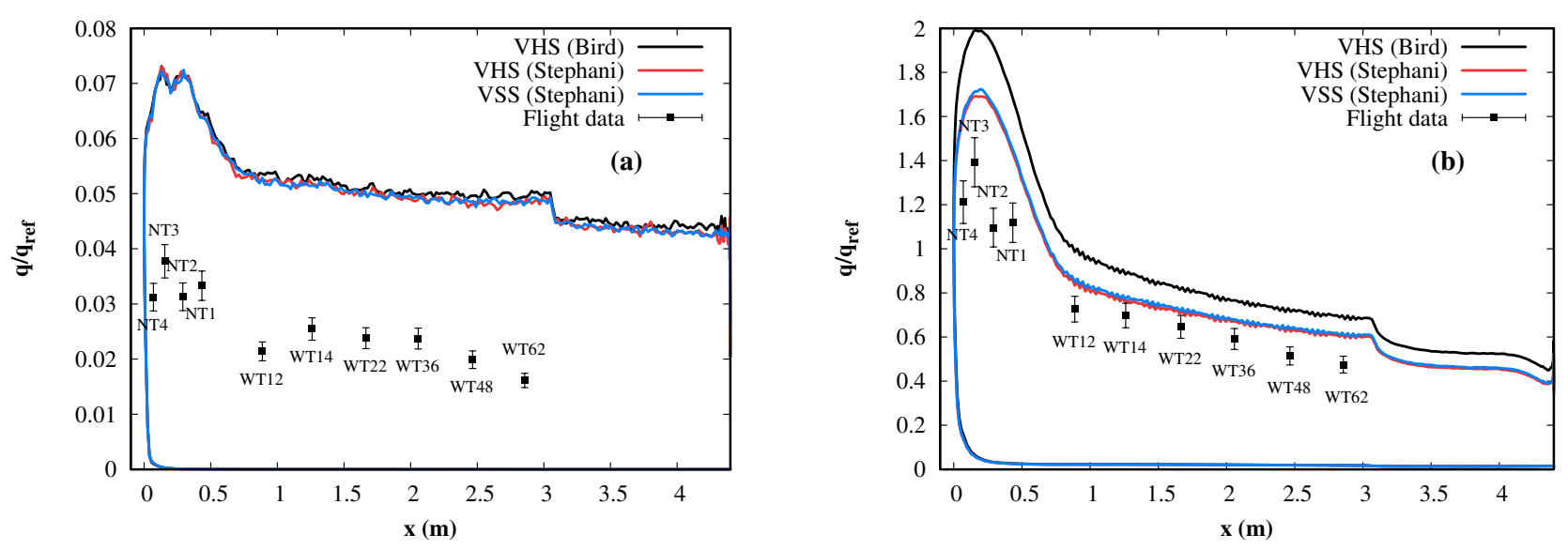

FigURE 26: Influence of the collision model on the DSMC heat flux distribution along the vehicle windward centerline at 115 (a) and 90 (b) km. Comparison with flight data.

The results in Figure 26b show that the optimized VHS collision model is responsible for absolute heat flux differences comprised between 10 and $20 \mathrm{~kW} / \mathrm{m}^{2}$ respectively in the windward and in the nose regions. In terms of relative difference, the optimized VHS collision model yields a heat flux reduction of $20 \%$ in the windward region and $13 \%$ in the nose region. Similarly to what was observed for the field quantities, the two optimized collision models give very close results in terms of heat flux. At $115 \mathrm{~km}$, all collision models give similar heat flux which is coherent with the fact that at high altitudes, the molecular interactions are dominated by the gas-surface interactions (Figure 26a).

Although the heat flux obtained at $90 \mathrm{~km}$ with Stephani's parameters is much closer to the measurements than the heat flux obtained with the standard VHS model, the collision model alone does not explain the discrepancies between the numerical results and the flight data. Indeed, the collision model has a small influence at $115 \mathrm{~km}$ and even at $90 \mathrm{~km}$, the relative difference with respect to the measurements are between 15\% (WT36) and 23\% (NT3). Moreover, the best-fit procedure of Stephani [35] was performed between 1000 and $5000 \mathrm{~K}$ and at $2300 \mathrm{~Pa}$ which does not exactly correspond to the considered conditions. This suggests that better results might be obtained with a better set of parameters. Over a wider range of temperature the fitting may however be challenging since the VHS and VSS parameters do not depend on the temperature [20]. Higdon et al. proposed in [44] another method to infer the VHS parameters of $\mathrm{H}_{2}$ and $\mathrm{He}$ by using collision integral parameters and over a wide range of temperature : $T \in[100 ; 10000] \mathrm{K}$.

\subsubsection{Sensitivity analysis to the free stream conditions}

Finally, the sensitivity to the free stream density was evaluated. The results of these computations are given in Figure 27.

For the highest altitude the heat flux is roughly proportional to the density as predicted by the free molecular theory $[19,20]$. Hence at $115 \mathrm{~km}$, the heat flux obtained with $0.85 \times \rho_{\infty}$ is $15 \%$ smaller. For the lowest altitude, the theory predicts a dependence of the heat flux proportional to the square root of the density [45]. In this case at $90 \mathrm{~km}$, the heat flux obtained with $0.85 \times \rho_{\infty}$ is $6 \%$ smaller. Hence, similarly to the accommodation coefficient, the density plays a major role in the heat flux prediction mostly at high altitudes. However, even if its influence and the uncertainty on its value both decrease as the altitude diminishes, above $85 \mathrm{~km}$, the deviation between the estimated density and its true value is likely to be greater than $15 \%$ and can therefore induce a significant change in the heat flux.

The analysis of the DSMC results evidenced large discrepancies with the flight data. Among the investigated sources of difference, the accommodation coefficient and the free stream density 

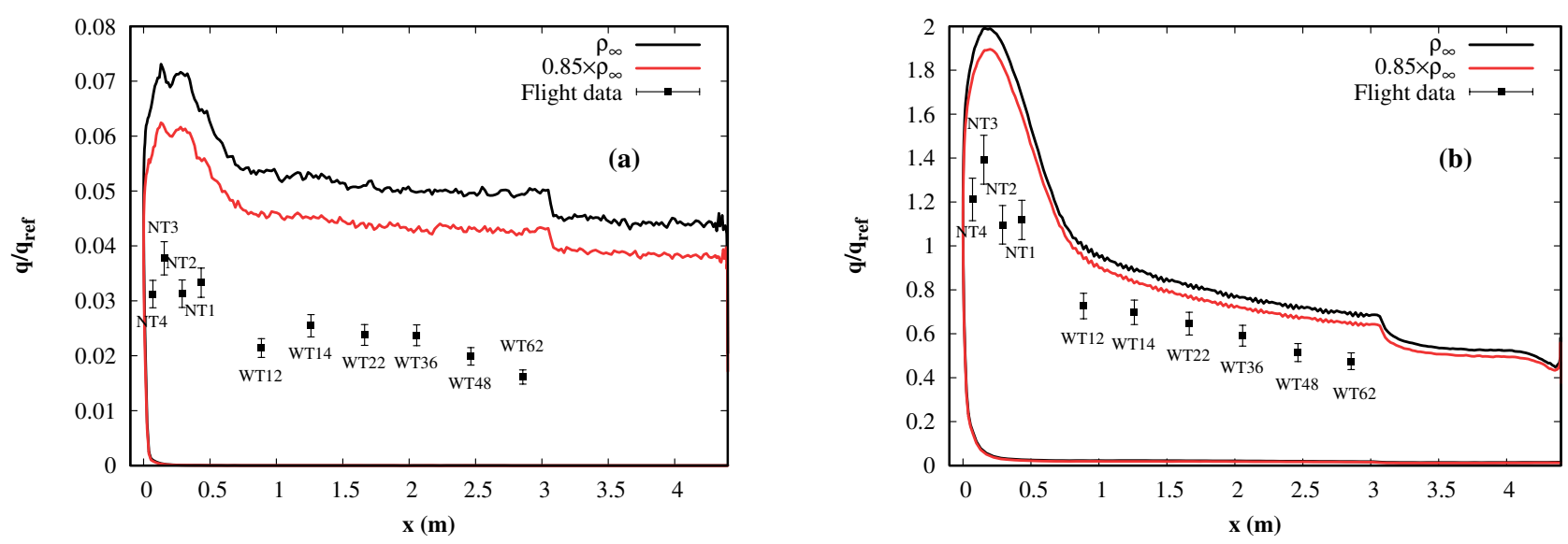

FIGURE 27: Influence of a 15\% free stream density uncertainty on the DSMC heat flux distribution along the vehicle windward centerline at 115 (a) and 90 (b) km. Comparison with flight data.

proved to have a considerable impact on the heat flux but to a lesser extent at low altitudes. The results also showed that the chemical factors have a small influence on the computed heat flux which is noticeable in the nose region only. For the lowest altitudes, using collision models with optimized parameters happens to drive the computed heat flux significantly closer to the measurements, while at $115 \mathrm{~km}$ sensibly the same results were obtained with all collision models. An uncertainty slightly greater than $15 \%$ on the free stream density combined with the use of an adequately parameterized collision model may lead to an even better agreement for the lowest altitudes.

\section{CFD versus DSMC results analysis}

At $90 \mathrm{~km}$, the Knudsen number reaches a value of $3.3 \times 10^{-3}$ which seems close enough to the continuum limit for the DSMC results to be compared to CFD computations. In order to better understand the differences between the DSMC results and the measurements, a CFD simulation was then performed. Unless specified otherwise, the numerical results are those obtained in the standard conditions introduced in section 3 .

\subsection{DSMC and CFD flowfield comparison at $90 \mathrm{~km}$}

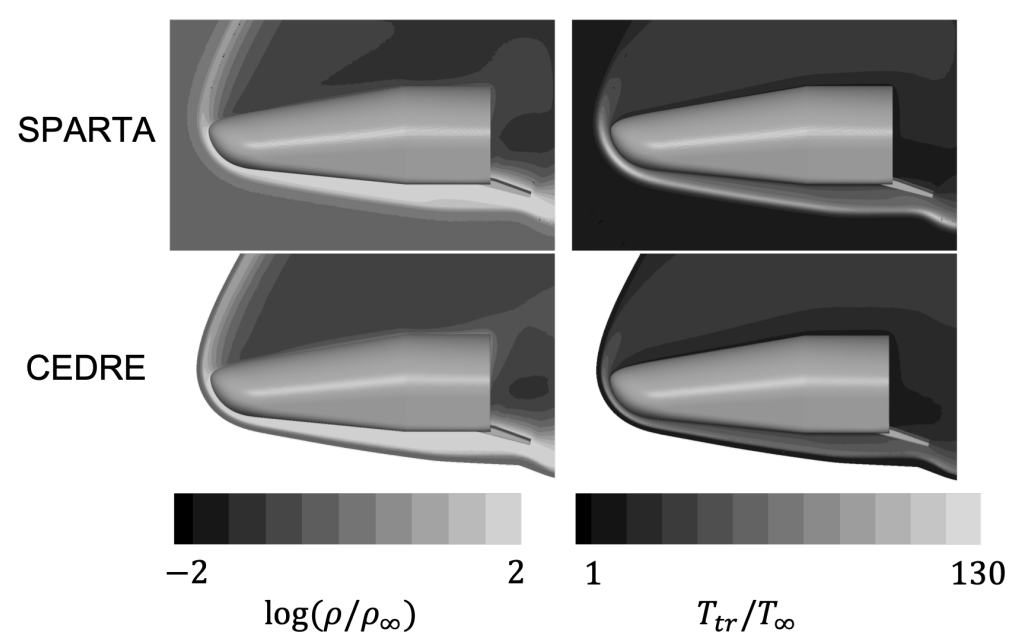

Figure 28: DSMC and CFD contours of normalized density (left) and translational temperature (right) at $90 \mathrm{~km}$.

The comparison of the CFD and DSMC results at $90 \mathrm{~km}$ is given in Figure 28. From these results, the following points can be noted : 
- The position of the shock layer predicted by the CFD calculations is closer to the wall than that predicted by the DSMC results.

- The thickness of the shock layer predicted by the CFD calculations is far smaller than that of the DSMC results.

- The peak of temperature in the shock is much smaller for the CFD results than for the DSMC ones.

- In the shadow region of the vehicle, the trends of the three contours is in relatively good agreement in spite of the significant rarefaction level of this area.

Because of the thermal equilibrium assumption and the unique temperature $T$ in CEDRE, one might question the relevance of comparing this temperature to the DSMC translational temperature (Figure 28) as well as to the translational and internal temperatures (Figure 29a). Thus, one can define the DSMC overall temperature $T_{\text {ov }}$ as follows :

$$
T_{\mathrm{ov}}=\frac{3 T_{\mathrm{tr}}+2 T_{\mathrm{rot}}+2 T_{\mathrm{vib}}}{7}
$$

and compare $T$ to $T_{\mathrm{ov}}$ instead. The comparison of $T$ and $T_{\mathrm{ov}}$ is given in Figure $29 \mathrm{~b}$ and shows a difference of around $14 \%$ between the maximal values which seems satisfying. However, the comparison of these temperatures profiles also indicates a relative difference in the shock layer distance and shock thickness of respectively 30 and 50\% with respect to the DSMC solution.

From a chemical perspective, Figure 29c shows that although the composition amplitudes of the DSMC computations are closer to the CFD results near the wall, significant discrepancies are still noticed along the control line. To make sure this was not due to the fact that the recombination reactions are allowed with CEDRE, a simulation preventing them was performed. The exact same compositions were obtained indicating that for the pressure conditions encountered at $90 \mathrm{~km}$, recombination reactions do not occur. The chemical composition differences are thought to be mostly due to the difference in the shock layer profiles (see Figures 29b and 29d). In addition, Morsa et al. [46] observed significant discrepancies between the DSMC and CFD chemical compositions and heat flux with the same chemical model. They attributed this deviation to the methodologies for handling the chemical processes that are different for both approaches.

Finally, Figure 29a shows the significant thermal nonequilibrium visible in the DSMC results where the internal temperatures remain much smaller than the translational temperature throughout the shock. In addition, the significant continuum breakdown areas are highlighted in Figure 30, where the $K n_{\mathrm{GLL}}$ contours of the CFD results reach values greater than 0.05 in the shock, near the wall and in the vehicle's shadow area. This observation is in total contradiction with the thermal equilibrium assumption of the CFD code. The use of the Navier-Stokes equations in these conditions is then questionable and the inability to accurately simulate the regions of significant thermochemical nonequilibrium could explain the large difference in the predicted shock position and thickness. However, both zoomed areas in Figures 29a and 29d show a good agreement between both methods in the near-wall region with similar velocity and temperature values.

A wide quantity of work devoted to the investigation of the continuum breakdown and to the comparison of CFD and DSMC solutions is reported in the literature and examples are given in Table 6. The first reference [47] shows that with the appropriate slip conditions in the case of a non-reacting and mono-species flow around a simple object (sphere and cylinder), a good agreement can be reached between the two solutions for a wide range of Knudsen number. In [33], the authors simulate a hypersonic flow around a 2D cylinder. They show that for a sufficiently low Knudsen number and when the DSMC collision model is calibrated in a consistent fashion with the CFD transport models, both solutions compare very favorably. In the same way but for a greater Knudsen number, Casseau's work [42] shows that for a simple reacting flow around 


\begin{tabular}{ccccccc}
\hline \hline Authors & CFD solver & DSMC solver & Flow & $M_{\infty}$ & $K n$ & Ref. \\
\hline Holman \& Boyd & LeMANS & MONACO & $\mathrm{N}_{2}$ non-reacting & 10 to 45 & 0.002 to 0.25 & {$[47]$} \\
Votta et al. & H3NS & DS2V & 5-air reacting & 28 & $0.002-0.01$ & {$[48]$} \\
Casseau et al. & hy2Foam & dsmcFoam & $\mathrm{N}_{2}$ reacting & 20 & 0.002 & {$[42]$} \\
Liechty et al. & LAURA & MAP & 2, 5, 11-air chemically frozen & $10-25$ & 0.0005 & {$[33]$} \\
\hline \hline
\end{tabular}

TABLE 6: Some CFD-DSMC comparison references in literature.

a 2D cylinder, both solutions can reach a satisfactory agreement. However, the consideration of a reacting 5 species air flow around a more complex geometry (axisymmetric probe) [48] led to more significant discrepancies between the computed flowfield solutions (difference in the shock thickness) except in the near-wall region.

In summary, these studies show that both methods can be brought in good agreement if the Knudsen number is sufficiently small and if the flow as well as the geometry are sufficiently simple. In the case of a fully reacting flow with more than 2 species and with complex geometries, the CFD solution converges by design towards a falsely continuous solution with a thin shock layer located very close to the body.

\subsection{Aerodynamic results analysis}

In spite of the differences that were raised previously, Figure 31 shows the excellent agreement between SPARTA and CEDRE with respect to the pressure coefficient distribution along the centerline. Concerning the skin friction coefficient, a relative difference between 20 and $30 \%$ is shown along the windward but an excellent agreement is visible in the nose region.
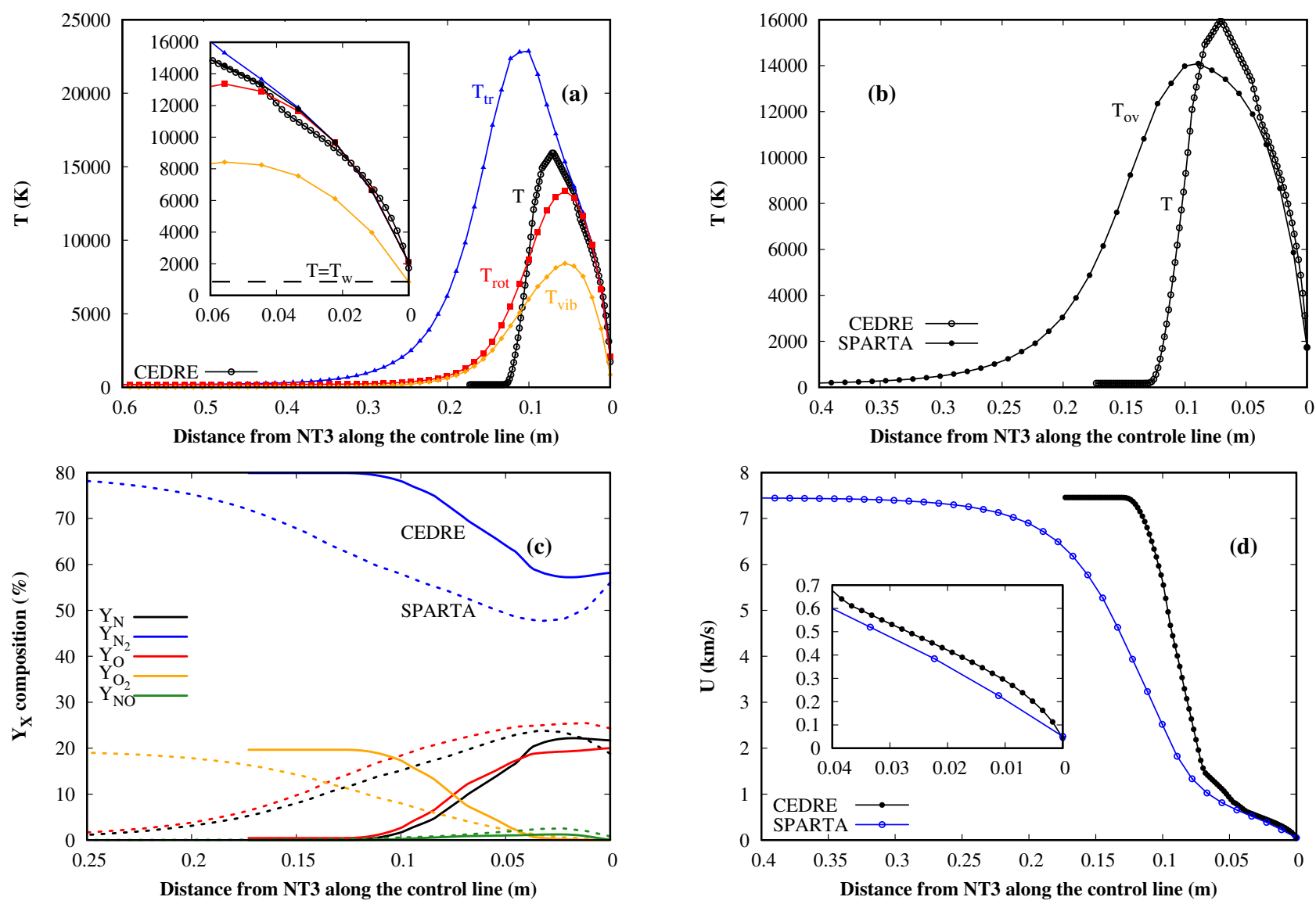

Figure 29: CFD versus DSMC temperature (a)-(b) chemical composition (c) and velocity (d) profiles along the control line at $90 \mathrm{~km}$. 


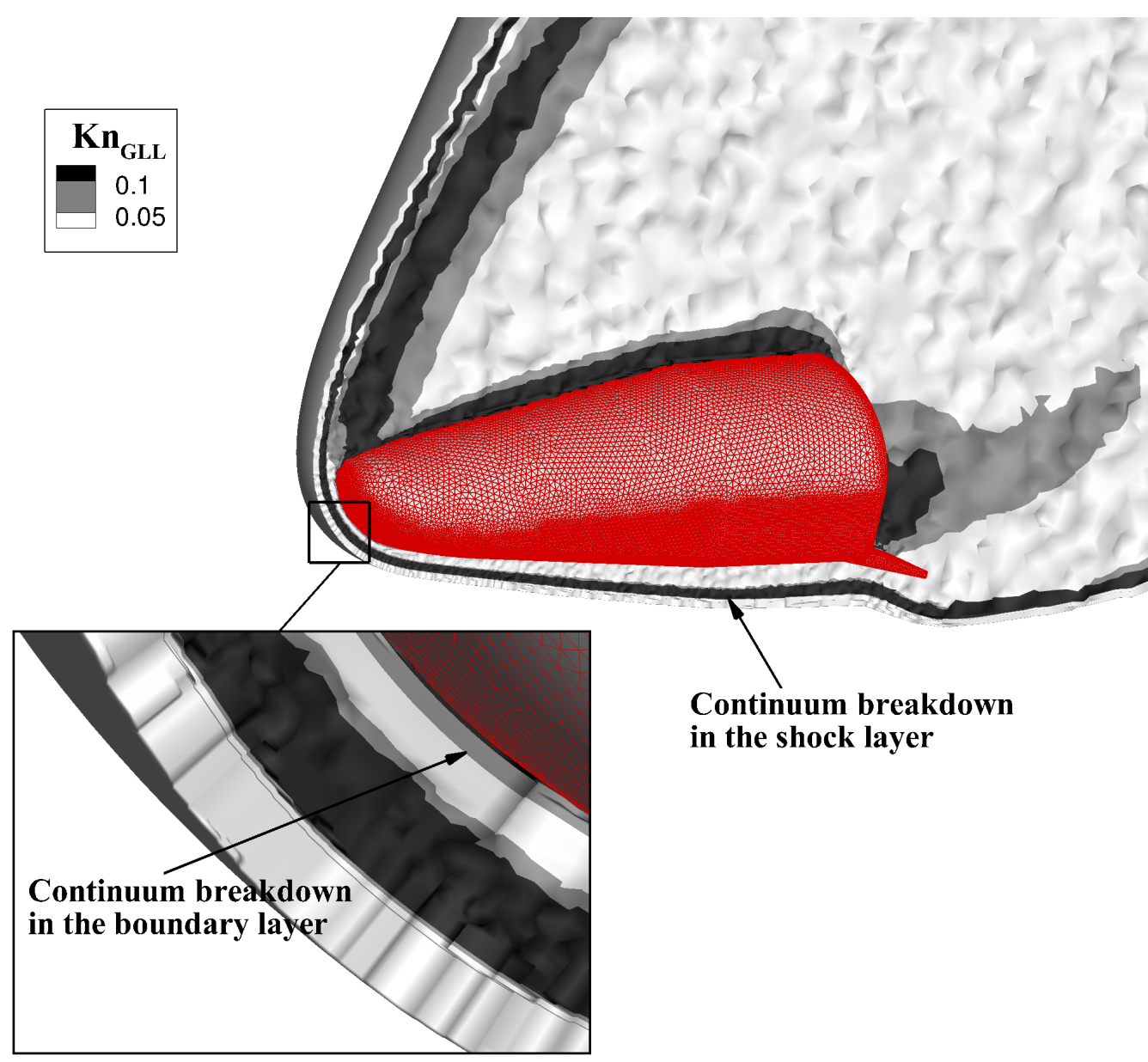

Figure 30: $K n_{\mathrm{GLL}}$ field for the CFD solution. In the light gray regions, $K n_{\mathrm{GLL}}<0.05$, in the gray regions $0.05<K n_{\mathrm{GLL}}<0.1$ and in the black regions $K n_{\mathrm{GLL}}>0.1$.
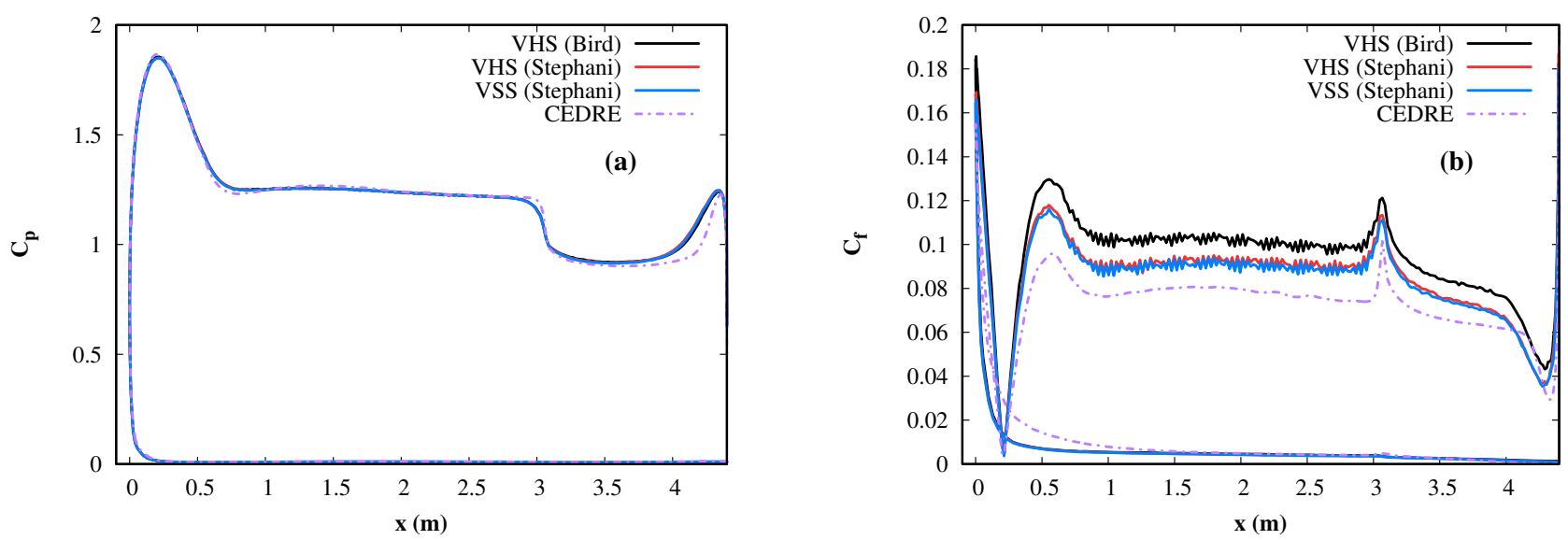

FiguRE 31: CFD vs DSMC pressure (a) and skin friction (b) coefficient distributions along the vehicle windward centerline at $90 \mathrm{~km}$.

Since the contribution of the skin friction to the aerodynamic coefficients is negligible compared to the pressure, a good agreement was still reached. Indeed, for the normal force coefficient and the axial force coefficient, relative differences of around $2 \%$ were observed between both solvers. For the pitching moment coefficient, a relative difference of $10 \%$ was obtained (see Table 7 ). In addition to the fact that the pitching moment coefficient is a more sensitive quantity, this difference might be explained by the discrepancy in the normal pressure distribution at the bottom of the windward and in the flap area (see Figure 32). With the collision models based on Stephani's 
parameters, similar pitching moment and normal coefficients are obtained. In comparison to the CFD results, the VHS model gives a better agreement for the axial force coefficient while the the discrepancy is slightly larger with the VSS model. Unfortunately, no experimental values are available for these aerodynamic quantities.

\begin{tabular}{cccc}
\hline \hline Solver & $C_{A}$ & $C_{N}$ & $C_{\mathrm{m}, \mathrm{cg}}$ \\
\hline SPARTA VHS (Bird) & -0.296 & 0.882 & -0.05 \\
SPARTA VHS (Stephani) & -0.284 & 0.877 & -0.05 \\
SPARTA VSS (Stephani) & -0.309 & 0.877 & -0.051 \\
CEDRE & -0.290 & 0.896 & -0.055 \\
\hline \hline
\end{tabular}

TABLE 7: CFD and DSMC aerodynamic coefficients at $90 \mathrm{~km}$.
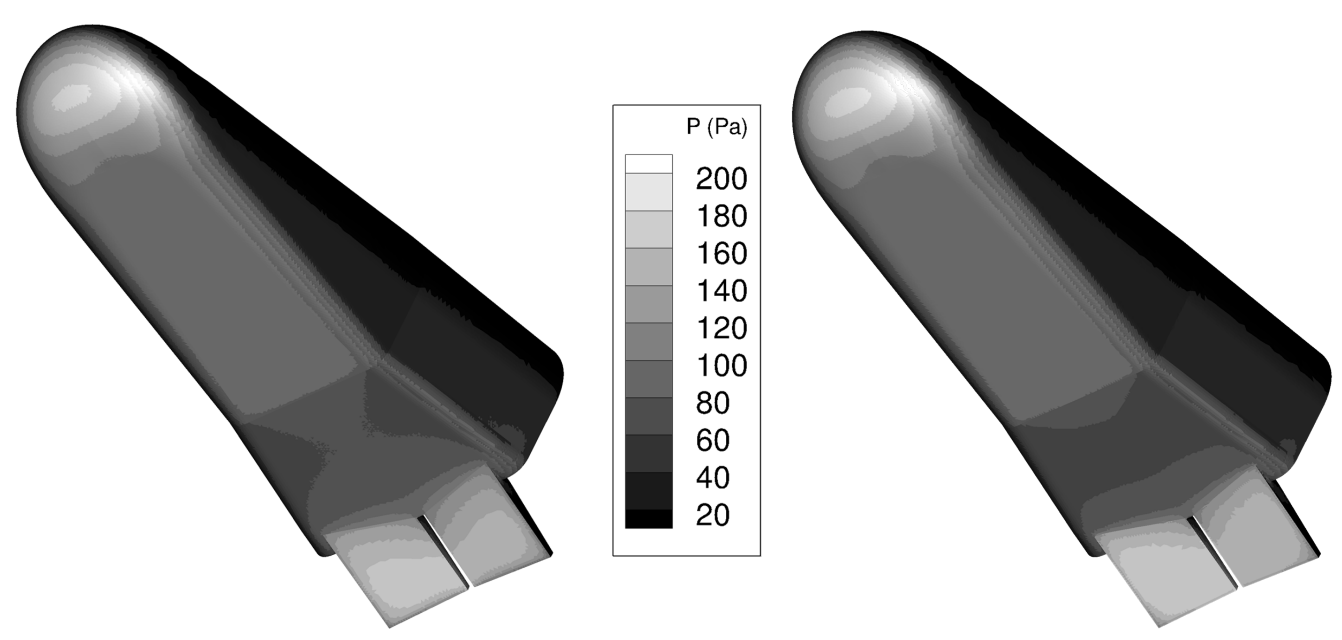

Figure 32: Comparison of the DSMC (left) and CFD (right) pressure contours on the vehicle windward at $90 \mathrm{~km}$.

\subsection{Aerothermodynamic results analysis}

Figure 33 shows the comparison of the flight data with the DSMC and CFD computations. In the nose region (Figure 33a), the relative difference between the flight data and the CFD results is of $10 \%$ only, while the relative difference between CEDRE and the standard SPARTA computations is of $30 \%$. With respect to the computations based on Stephani's parameters, a much better agreement is observed between the two methods and a difference of around $14 \%$ only is obtained in the nose region. Going away from the centerline (Figures 33b to 33d), the gap between the two methods decreases significantly and a good agreement is reached in the side and leeward regions.

In comparison to the flight data, the CFD results are surprisingly good considering the limitations pointed out in section 5.1. The object of this section is then to understand why the discrepancies between both solvers vary that much and whether such behavior should be expected or not.

In terms of models differences between the two solvers, the major drawback of CEDRE is that it is not provided with a vibrational temperature. It is quite complicated to quantify how the addition of such functionality would affect the results but the energy conservation principle suggests that adding an internal mode of energy should only weakly change the wall heat flux. This was confirmed with a DSMC computation in which the vibrational mode of energy was not activated. At $90 \mathrm{~km}$, this simulation yielded a wall heat flux $3 \%$ smaller than that of the standard 

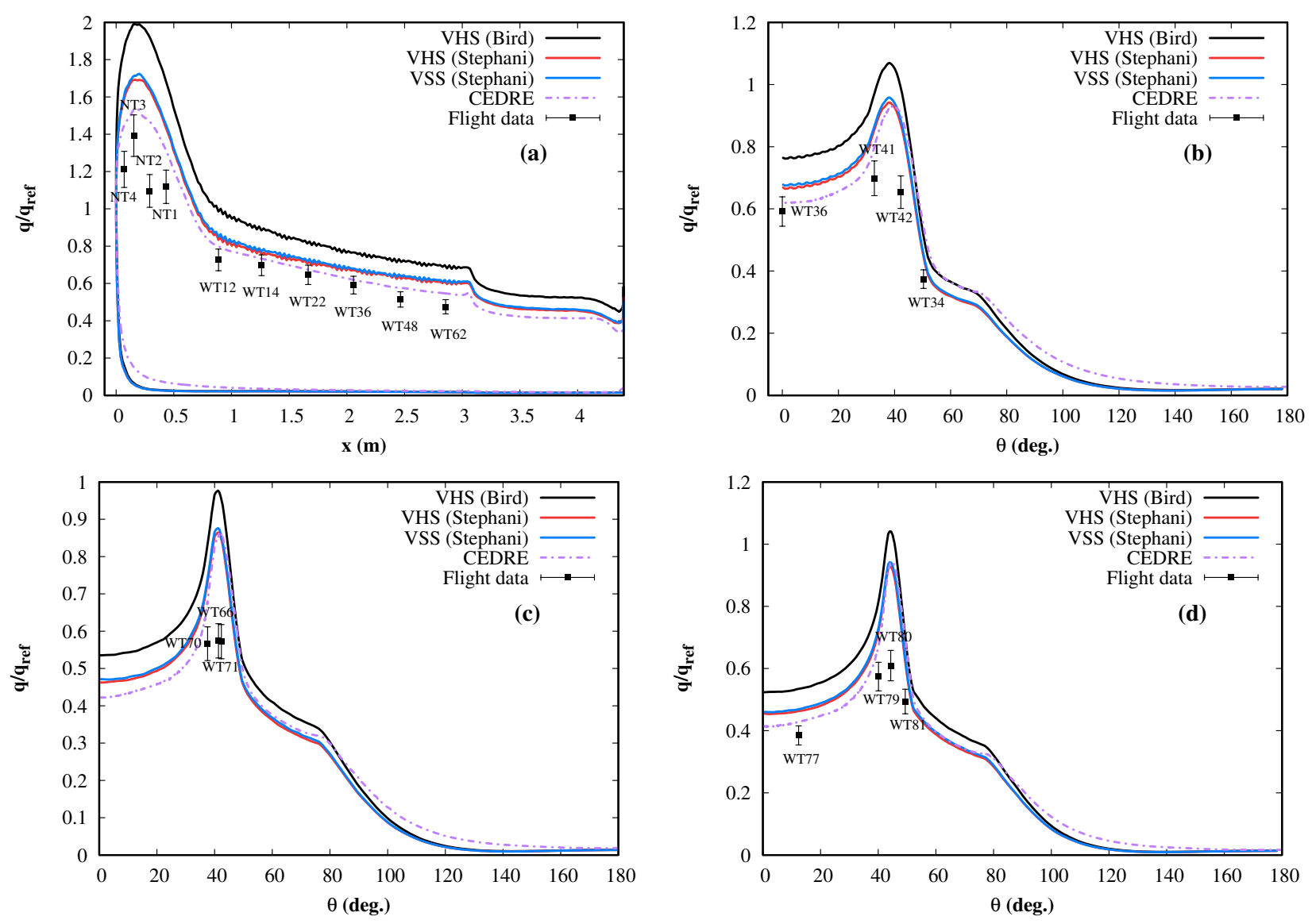

FIGURE 33: Comparison of the flight data, the CFD and DSMC heat flux distributions along the vehicle windward centerline (a), at $x=2.06 \mathrm{~m} \mathrm{(b),} \mathrm{at} x=3.52 \mathrm{~m} \mathrm{(c)}$ and at $x=3.99 \mathrm{~m} \mathrm{(d)}$ at $90 \mathrm{~km}$.

conditions. Hence, the thermal equilibrium assumption in the CFD solver does not explain the heat flux deviation between both methods but it could still slightly improve their agreement.

Furthermore, in CFD solvers, the convective heat flux is obtained from Fourier's law which gives the expression of its conductive part and Fick's law which defines the expression of its diffusive part. Mathematically, the wall heat flux $q$ is written :

$$
q=\underbrace{\left(\kappa_{\text {mix }} \partial_{x} T\right)_{w}}_{\text {conductive part }}+\underbrace{\left(\sum_{i} \rho D_{i} h_{i} \partial_{x} C_{i}\right)_{w},}_{\text {diffusive part }}
$$

where $\kappa_{\text {mix }}$ is the mixture conductivity, $\partial_{x} T$ is the temperature gradient, $D_{i}$ is the $i^{\text {th }}$ component of the mixture molecular diffusion coefficient, $h_{i}$ is the enthalpy of species $i$ and $\partial_{x} C_{i}$ is the gradient of the species $i$ mass fraction.

For a non-catalytic boundary condition, the diffusive part cancels out and the convective heat flux is simply given by its conductive part. Equation (11) then suggests that for a given thermal gradient, the conductive heat flux only depends on the mixture conductivity which usually depends on the species viscosity and conductivity as well as on the mixture viscosity. All these parameters can be computed with various approximations.

In [47], the authors modified the CFD solver according to the following equations :

$$
\begin{gathered}
\mu=\mu_{\mathrm{ref}}\left(\frac{T}{T_{\mathrm{ref}}}\right)^{\omega}, \\
\mu_{\mathrm{ref}}=\frac{15 \sqrt{\pi m k T_{\mathrm{ref}}}}{2 \pi d_{\mathrm{ref}}^{2}(5-2 \omega)(7-2 \omega)},
\end{gathered}
$$


where $\omega, T_{\text {ref }}$ and $d_{\text {ref }}$ are the parameters of the VHS model employed in the DSMC computations. This modification forces the CFD solver to use a viscosity consistent with that of the DSMC computations and most likely explains the excellent agreement between LeMans and MONACO in terms of heat flux coefficient for Knudsen numbers ranging from 0.002 to 0.05 .

In [33], Liechty et al. compared the DSMC and CFD mixture viscosity and conductivity in the Chapman-Enskog framework. For their computations, the CFD transport models are based on $a b$ initio data and the DSMC stagnation heat flux is greater than that of CFD computation by $5 \%$ (VSS model) and 10\% (VHS model) for the Mach 25 case.

In [42], the species viscosity and conductivity are respectively computed with Blottner and Eucken's formula while the mixture quantities are obtained with the Armally-Sutton mixing law. With these models, the stagnation heat flux of the CFD computations is greater than that of the DSMC by values comprised between 20 and $30 \%$.

Finally, in [48], Votta employed Yun and Mason collision integrals for the computation of the species viscosity, Eucken's formula for the species conductivity and the mixture quantities were computed with Wilke's formula. For their computations at $85 \mathrm{~km}$, their CFD solver gave a wall heat flux distribution around $60 \%$ smaller than the DSMC solution for the non-catalytic case. For the fully-catalytic computations the relative difference was of only $10 \%$. However, for both boundary conditions, the authors noted that the absolute difference in the heat flux distribution was approximately the same (between 30 and $40 \mathrm{~kW} / \mathrm{m}^{2}$ ) confirming that the gap between both methods lies in the conductive part of the heat flux.

In CFD, there are a large number of models for the computation of the species and mixture transport properties. These models can assume different kind of relationships between the species and mixture quantities which are determining for the computation of the mixture conductivity and ultimately for the computation of the heat flux. Reddy and Sinha [49] investigated the influence of the species viscosity model on the surface heat flux of Fire II at $35 \mathrm{~km}$. Their results showed a slight increase in the shock stand-off distance and a $10 \%$ increase in the wall heat flux with a collisionintegral based species viscosity instead of the Blottner model. In the same way, Palmer and Wright [50] evidenced significant variations of mixture viscosity from one model to another as the temperature increases for an 11-air mixture at $100 \mathrm{kPa}$. With Eucken's formula for instance, the mixture conductivity is directly proportional to the mixture viscosity. In this case, an important change in the mixture viscosity yields the same change in the mixture conductivity and in the heat flux. In addition, as explained by Boyd in [20], Eucken's formula is known to gain in accuracy in the near-equilibrium limit but for the simulation of a rarefied flow presenting a significant degree of continuum breakdown, one might question the applicability of such approximation.

In conclusion, among the various transport quantities approximations, their applicability to the simulation of a rarefied flow is a major concern for ensuring the computation of a consistent heat flux. In addition, a rapid examination of two references evidenced the large sensitivity of the transport properties and more generally of the heat flux to the chosen approximations and leads to think that for the same free stream conditions, non-negligible heat flux deviations could be obtained with the same CFD solver.

\section{Conclusions}

For the first time, a post-flight reconstruction of the IXV rarefied portion of the reentry was performed with the DSMC code SPARTA between 115 and $90 \mathrm{~km}$. At $90 \mathrm{~km}$, CFD computations were also made with CEDRE, in order to compare both methods in the near-continuum limit. The IXV measurements constitute a unique mean of numerical evaluation through the consideration of the reconstructed heat flux at 20 locations on the vehicle.

First, the paper drew up an overview of the IXV mission through the description of the geometry and the trajectory of the vehicle as well as its instrumentation. Then, the results obtained 
with two numerical approaches (DSMC and CFD) were analyzed and compared to the available flight data. In this way, the impact of various numerical parameters was assessed. Among these parameters, the accommodation coefficient, the collision model and the chemical models were investigated. Finally, the effects of the free stream density uncertainty on the wall quantities was quantified.

The investigation of the flowfield highlighted several aspects of interest such as the rarefaction and accommodation coefficient effects on the shock structure and the decrease of the accommodation coefficient role as the altitude diminishes. At $90 \mathrm{~km}$, significant discrepancies were observed between both methods flowfield solutions (shock wave position and thickness) but coherent results were obtained in the near wall region. The main differences between the two methods are probably due to the significant amount of continuum breakdown at $90 \mathrm{~km}$ as well as to the differences of conductivity and thermal diffusivity.

Concerning the flight data, some high altitude sensors present anomalies bringing into question their reliability. Indeed, on the windward region, some thermocouples (WT62 and WT48) slowly recover over time which is probably due to the fact that at high altitudes, the wall temperature distribution is too close to the thermocouple minimal measurable value. For some other thermocouples (NT2 and WT12), the reported values are simply off for all altitudes which indicate a probable malfunction. In a general manner, the capacity of the sensors to measure the correct temperature is questioned. From a numerical perspective, the DSMC results showed the capacity of the method to partially approach the levels of the recorded heat flux above $105 \mathrm{~km}$ by using a non fully diffuse gas-surface interaction model but with two distinct accommodation coefficient values. Moreover, the influence of this parameter decreases with the altitude and cannot explain the global DSMC overestimation of the flight data. At 115, 100 and $95 \mathrm{~km}$ relative differences in the nose region are comprised between 90 and $110 \%$ and between 40 and $50 \%$ at 110, 105 and $90 \mathrm{~km}$. For the highest altitudes, the sensitivity analysis showed that a portion of the gap can be attributed to the uncertainty on the free stream density while using adequate parameters for the collision model enabled to considerably improve the numerical results at $90 \mathrm{~km}$. Concerning the chemical models, the results showed that the heat flux was weakly affected by this factor.

For the lowest flight point, a very good agreement between the CFD and the DSMC was obtained for the aerodynamic quantities but unfortunately, no flight data are available. With respect to the heat flux prediction, discrepancies of around respectively 30\% (Bird's VHS parameters) and 15\% (Stephani's VHS and VSS parameters) were observed between both methods in noncatalytic conditions and a good agreement was found between CEDRE and the measurements. Nevertheless, since for real conditions the wall is necessarily partially catalytic, the heat flux of the numerical solutions computed with a non-catalytic assumption is expected to be smaller than the measurements. Here, even the CFD solution which provided the smallest heat flux overestimates the measurements. Moreover, the analysis of the models for the transport quantities suggests that there is room for the CFD heat flux adjustment towards the DSMC solution. In this case, a better agreement between both methods would drive the CFD solution further away from the flight data. A significant uncertainty on the free stream density could be responsible for this disagreement. As for now, the DSMC method appears to provide a more conservative solution.

Finally, the IXV rarefied reentry is a challenging study case and this work raises many questions. First the instrumentation seems to be unreliable in highly rarefied conditions. It underlines, once more, the necessity to design high altitude flight experiments with adequate sensors. The sensitivity analysis also showed the need to perfectly know the atmospheric environment in order to only focus on the numerical models. Finally, complementary efforts must be carried in order to reach consistence between the two methods both for the flowfield and the surface quantities. 


\section{Acknowledgement}

This work was supported by the ONERA and DGA (Direction Générale de l'Armement), the French Ministry of Armed Forces, through the co-funding of the PhD of M. Schouler. This work was also granted access to the HPC resources of CALMIP under the allocation 2020 - Project 20018. ONERA HPC resources have also been used. The authors thank the European Space Agency, Thales Alenia Space Italy and Dassault Aviation for their support of this work, their flight data and the authorization to communicate them in a publication.

We also gratefully acknowledge Thierry Magin, Zuheyr Alsalihi and Federico Bariselli from the Von Karman Institute for useful discussions. We also thank Vincent Drouet from the ONERA for his help in designing the CFD mesh.

\section{References}

[1] E. Kustova, A. Krylov, V. Lashkov, M. Mekhonoshina, Experimental Facilities and Modelling for Rarefied Aerodynamics, Tech. rep., NATO (2011).

[2] P. Reynier, Survey of high-enthalpy shock facilities in the perspective of radiation and chemical kinetics investigations, Progress in Aerospace Sciences 85 (2016) 1-32. doi:10.1016/j.paerosci.2016.04.002.

[3] M. Schouler, Y. Prévereaud, L. Mieussens, Survey of flight and numerical data of hypersonic rarefied flows encountered in earth orbit and atmospheric reentry, Progress in Aerospace Sciences 118 (2020) -. doi: $10.1016 / j$.paerosci.2020.100638.

[4] R. Haya-Ramos, G. Blanco, I. Pontijas, D. Bonetti, J. Freixa, C. Parigini, E. Bassano, R. Carducci, M. Sudars, A. Denaro, R. Angelini, S. Mancuso, The design and realisation of the IXV Mission Analysis and Flight Mechanics, Acta Astronautica 124 (2016) 39-52. doi:10.1016/j.actaastro.2015.12.048.

[5] R. Angelini, A. Denaro, IXV re-entry demonstrator : Mission overview, system challenges and flight reward, Acta Astronautica 124 (2016) 18-30. doi:10.1016/j.actaastro.2016.02.015.

[6] L. Van Ghele, Analyse aérothermique de la rentrée atmosphérique de l'Intermediate eXperimental Vehicle (IXV), Ph.D. thesis, ISAE-SUPAERO (2020).

[7] T. Banyai, E. Torres, T. E. Magin, A. V. Kashkovsky, P. V. Vashchenkov, M. S. Ivanov, P. Rambaud, Evaluation of the aerodynamic properties of the intermediate experimental vehicle in the rarefied and transitional regime, Progress in Flight Physics 5 (2013) 425-440. doi:10.1051/eucass/201305425.

[8] S. J. Plimpton, S. G. Moore, A. Borner, A. K. Stagg, T. P. Koehler, J. R. Torczynski, M. A. Gallis, Direct simulation Monte Carlo on petaflop supercomputers and beyond, Physics of Fluids 31 (8) (2019) 1-18. doi: $10.1063 / 1.5108534$.

[9] M. Fumo, R. Scigliano, M. Belardo, G. C. Rufolo, A. Esposito, M. Linari, Aero-Thermal Post Flight Analysis of IXV Control Surfaces, in : 7Th European Conference for Aeronautics and Space Sciences (Eucass), no. February, 2015, pp. 1-11. doi:10.13009/EUCASS2017-262.

[10] J. L. Shinn, J. N. Moss, A. L. Simmonds, Viscous-Shock-Layer Heating Analysis for the Shuttle Windward Plane with Surface Finite Catalytic Recombination Rates, in : 3rd Joint Thermophysics, Fluids, Plasma and Heat Transfer Conference, 1982.

[11] G. M. Kelly, M. L. Heck, J. G. McConnell, L. A. Waters, P. A. Troutman, Challenger STS-17 (41-G) PostFlight Best Estimate Trajectory Products - Development and Summary Results, Tech. rep., NASA (1987).

[12] F. Buffenoir, C. Zeppa, T. Pichon, F. Girard, Development and flight qualification of the C-SiC thermal protection systems for the IXV, Acta Astronautica 124 (2016) 85-89. doi:10.1016/j . actaastro.2016.02. 010.

[13] M. Cioeta, G. Di Vita, M. T. Signorelli, G. Bianco, M. Cutroni, F. Damiani, V. Ferretti, A. Rotondo, Design, qualification, manufacturing and integration of IXV Ablative Thermal Protection System, Acta Astronautica 124 (2016) 90-101. doi:10.1016/j.actaastro.2015.12.026.

[14] F. Buffenoir, D. Escafre, T. Brault, L. Rival, F. Girard, Dynamical and thermal qualification of the C-SiC nose for the IXV, Acta Astronautica 124 (2016) 79-84. doi:10.1016/j.actaastro.2016.02.008.

[15] G. C. Rufolo, F. Camarri, C. Pereira, R. Romano, D. Vernani, ESA intermediate experimental vehicle in-flight experimentation. Objectives, experiment implementation, qualification and integration, in : Proceedings of the International Astronautical Congress, IAC, Vol. 10, 2014, pp. 7488-7501. doi:10.13140/2.1.2093.8249.

[16] L. Van Ghele, P. Reulet, J.-L. Verant, P. Millan, J.-L. Battaglia, Aerothermal Analysis of the Atmospheric Reentry of the Intermediate eXperimental Vehicle (IXV), in : International Heat Transfer Conference 16, Begellhouse, Connecticut, 2018, pp. 2699-2706. doi:10.1615/IHTC16.cip.024117.

[17] L. Sagnieres, I. Sharf, Uncertainty Characterization of Atmospheric Density Models for Orbit Prediction of Space Debris, in : 7th European Conference on Space Debris, no. June, Darmstadt, 2017, pp. 18-21.

[18] Unknown, COSPAR International Reference Atmosphere (CIRA-2012), Tech. rep. (2012). 
[19] G. A. Bird, Molecular gas dynamics and the direct simulation of gas flows, Clarendon Press, 1994.

[20] I. D. Boyd, T. E. Schwartzentruber, Nonequilibrium gas dynamics and molecular simulation, Cambridge University Press, Cambridge, 2017. doi:10.1017/9781139683494.

[21] E. Josyula, J. Burt, Review of Rarefied Gas Effects in Hypersonic Applications, Tech. rep., NATO (2011). doi:10.1080/02690949508726249.

[22] T. E. Schwartzentruber, I. D. Boyd, Progress and future prospects for particle-based simulation of hypersonic flow, Progress in Aerospace Sciences 72 (2015) 66-79. doi:10.1016/j.paerosci.2014.09.003.

[23] A. Refloch, B. Courbet, A. Murrone, C. Laurent, J. Troyes, G. Chaineray, J. B. Dargaud, F. Vuillot, CEDRE Software, Journal AerospaceLab (2) (2011) 1-10.

[24] D. Enskog, Kinetische Theorie der Vorgänge in mässig verdünnten Gasen.

[25] I. D. Boyd, T. R. Deschenes, Hybrid particle-continuum numerical methods for aerospace applications, Tech. rep., NATO (2011).

[26] R. Probstein, Shock Wave and Flow Field Development in Hypersonic Re-Entry, ARS Journal 31 (2) (1961) 185-194. doi:10.2514/8.5423.

[27] J. F. Padilla, Assessment of Gas-Surface Interaction Models for Computation of Rarefied, Ph.D. thesis, The University of Michigan (2008).

[28] K. Moe, M. M. Moe, Gas-surface interactions in low-Earth orbit, in : AIP Conference Proceedings, Vol. 1333, 2011, pp. 1313-1318. doi:10.1063/1.3562825.

[29] N. H. Crisp, P. C. Roberts, S. Livadiotti, A. Macario Rojas, V. T. Oiko, S. Edmondson, S. J. Haigh, B. E. Holmes, L. A. Sinpetru, K. L. Smith, J. Becedas, R. M. Domínguez, V. Sulliotti-Linner, S. Christensen, J. Nielsen, M. Bisgaard, Y. A. Chan, S. Fasoulas, G. H. Herdrich, F. Romano, C. Traub, D. García-Almiñana, S. Rodríguez-Donaire, M. Sureda, D. Kataria, B. Belkouchi, A. Conte, S. Seminari, R. Villain, In-orbit aerodynamic coefficient measurements using SOAR (Satellite for Orbital Aerodynamics Research), Acta Astronautica 180 (2021) 85-99. doi:10.1016/j.actaastro.2020.12.024.

URL https://doi.org/10.1016/j.actaastro.2020.12.024

[30] J. N. Moss, G. A. Bird, Direct Simulation of Transitional Flow for Hypersonic Reentry Conditions, in : AIAA, 1988, pp. 830-843. doi:10.2514/2.6909.

[31] S. F. Gimelshein, I. J. Wysong, Bird's total collision energy model : 4 decades and going strong, Physics of Fluids 31 (7) (2019) 1--16. doi:10.1063/1.5097706.

[32] K. Koura, H. Matsumoto, Variable soft sphere molecular model for inverse-power-law or Lennard-Jones potential, Physics of Fluids A : Fluid Dynamics 3 (10) (1991) 2459-2465. doi:10.1063/1.858184.

[33] D. S. Liechty, A. J. Wise, S. Subramaniam, K. A. Stephani, Comparison of CFD and DSMC using calibrated transport parameters, in : AIP Conference Proceedings, Vol. 2132, 2019. doi:10.1063/1.5119570.

[34] K. Swaminathan-Gopalan, K. A. Stephani, Recommended direct simulation Monte Carlo collision model parameters for modeling ionized air transport processes, Physics of Fluids 28 (2). doi:10.1063/1.4939719.

[35] K. A. Stephani, D. B. Goldstein, P. L. Varghese, Consistent treatment of transport properties for five-species air direct simulation Monte Carlo/Navier-Stokes applications, Physics of Fluids 24 (7) (2012) 1--23. doi: $10.1063 / 1.4729610$.

[36] T. K. Mankodi, U. V. Bhandarkar, B. P. Puranik, Hypersonic flow over Stardust Re-entry Capsule using abinitio based chemical reaction model, Acta Astronautica 162 (2019) 243-255. doi:10.1016/j.actaastro. 2019.06 .021$.

[37] G. A. Bird, T. Abe, A Comparison of Collision Energy-based and Temperature-based Procedures in DSMC, in : AIP Conference Proceedings, Vol. 1084, AIP, 2009, pp. 245-250. doi:10.1063/1.3076480.

[38] G. A. Bird, The Q-K model for gas-phase chemical reaction rates, Physics of Fluids 23 (10) (2011) 1-13. doi:10.1063/1.3650424.

[39] G. A. Bird, Chemical reactions in DSMC, in : AIP Conference Proceedings, Vol. 1333, American Institute of Physics, 2011, pp. 1195-1202. doi:10.1063/1.3562806.

[40] M. A. Gallis, R. B. Bond, J. R. Torczynski, Assessment of collisional-energy-based models for atmospheric species reactions in hypersonic flows, Journal of Thermophysics and Heat Transfer 24 (2) (2010) 241-253. doi:10.2514/1.46267.

[41] M. A. Gallis, R. B. Bond, J. R. Torczynski, A kinetic-theory approach for computing chemical-reaction rates in upper-atmosphere hypersonic flows, Journal of Chemical Physics 131 (12) (2009) 1-13. doi:10.1063/1. 3241133.

[42] V. Casseau, D. E. R. Espinoza, T. J. Scanlon, R. E. Brown, A two-temperature open-source CFD model for hypersonic reacting flows, part two : Multi-dimensional analysis, Aerospace 3 (4) (2016) 1-15. doi: 10.3390/aerospace3040045.

[43] X. Y. Wang, C. Yan, Y. K. Zheng, E. L. Li, Assessment of chemical kinetic models on hypersonic flow heat transfer, International Journal of Heat and Mass Transfer 111 (2017) 356-366. doi:10.1016/j. ijheatmasstransfer.2017.03.102.

[44] K. J. Higdon, B. A. Cruden, A. M. Brandis, D. S. Liechty, D. B. Goldstein, P. L. Varghese, DSMC shock 
simulation of saturn entry probe conditions, in : 46th AIAA Thermophysics Conference, 2016, pp. 1-18. doi:10.2514/6.2016-3840.

[45] K. Sutton, A. J. Graves, A general stagnation-point convective-heating equation for arbitrary gas mixtures, Tech. Rep. November 1971, NASA (1971).

[46] L. Morsa, G. Zuppardi, R. Votta, A. Schettino, Influence of chemical models on heat flux for EXPERT and Orion capsules, in : Proceedings of the Institution of Mechanical Engineers, Part G : Journal of Aerospace Engineering, Vol. 228, 2014, pp. 930-948. doi:10.1177/0954410013483935.

[47] T. D. Holman, I. D. Boyd, Effects of continuum breakdown on the surface properties of a hypersonic sphere, Journal of Thermophysics and Heat Transfer 23 (4) (2009) 660-673. doi:10.2514/1.43509.

[48] R. Votta, A. Schettino, G. Ranuzzi, S. Borrelli, Hypersonic low-density aerothermodynamics of Orion-like exploration vehicle, Journal of Spacecraft and Rockets 46 (4) (2009) 781-787. doi:10.2514/1.42663.

[49] D. S. K. Reddy, K. Sinha, Effect of Transport Coefficients on Aero-thermal Predictions of Re-entry Flows, in : Symposium on Applied Aerodynamics and Design of Aerospace Vehicle, 2009. doi:10.2514/1.41380.

[50] G. E. Palmer, M. J. Wright, Comparison of Methods to Compute High-Temperature Gas Viscosity, Journal of Thermophysics and Heat Transfer 17 (2) (2003) 232-239. doi:10.2514/6.2002-3342. 